\title{
Metalogenia del depósito de manganeso Santa Rosa, Baja California Sur, México
}

\author{
Augusto Antonio Rodríguez-Díaz ${ }^{1,2^{*}}$, David Blanco-Florido ${ }^{1}$, Carles Canet ${ }^{3}$, \\ Fernando Gervilla-Linares ${ }^{4}$, Eduardo González-Partida ${ }^{5}$, Rosa María Prol-Ledesma ${ }^{3}$, \\ Salvador Morales-Ruano ${ }^{4}$, Maite García-Vallès ${ }^{6}$
}

\footnotetext{
${ }^{1}$ Posgrado en Ciencias de la Tierra, Universidad Nacional Autónoma de México, Ciudad Universitaria, 04510, México, D.F. ${ }^{2}$ Facultad de Ciencias de la Tierra, Universidad Autónoma de Nuevo León, Ex-Hacienda de Guadalupe, Carretera LinaresCerro Prieto km 8, 67700, Linares, Nuevo León, México.

${ }^{3}$ Instituto de Geofísica, Universidad Nacional Autónoma de México, Ciudad Universitaria, 04510, México, D.F.

${ }^{4}$ Departamento de Mineralogía y Petrología e Instituto Andaluz de Ciencias de la Tierra, Universidad de Granada, Avd. Fuentenueva s/n., 18002, Granada, España.

${ }^{5}$ Centro de Geociencias, Universidad Nacional Autónoma de México, Blvd. Juriquilla 3001, Juriquilla, Querétaro, 76230 , México

${ }^{6}$ Departament de Cristal.lografia, Mineralogía i Dipòsits Minerals, Universitat de Barcelona, c. Martí i Franquès s.n., Barcelona 08028, Catalunya, España.

*geaard@hotmail.com
}

\section{Resumen}

El depósito Santa Rosa (Mn-Ba) se encuentra localizado al SO del poblado de San Nicolás, al sur de la Península Concepción, Baja California Sur, México. Consiste en una veta-brecha de óxidos de manganeso de $500 \mathrm{~m}$ de longitud, expuesta en afloramientos discontinuos, con rumbo preferente NNO-SSE e inclinaciones subverticales hacia el ENE. Se encuentra encajonada en conglomerados del miembro Los Volcanes, perteneciente a la Formación San Nicolás del Plioceno. Su mineralización está formada mayoritariamente por estructuras bandeadas y brechadas con óxidos de manganeso (romanechita y pirolusita), barita, ópalo, cuarzo, calcita, magnetitamaghemita y goethita.

El contenido de manganeso de las menas varía entre 23.6 y $50.0 \%$ en peso de $\mathrm{MnO}$, mientras que el de $\mathrm{SiO}_{2}$ oscila entre 13.3 y $32.4 \%$ y el de $\mathrm{BaO}$ entre 9.9 y 13.7 \%. Además, se observa un enriquecimiento significativo en $\mathrm{Sr}, \mathrm{Sb}, \mathrm{As}, \mathrm{Cu}$ y $\mathrm{Zn}$. Los perfiles de elementos de tierras raras normalizados a NASC muestran una marcada anomalía negativa de Eu y una anomalía negativa menos intensa de Ce. Las relaciones de $\mathrm{Fe} / \mathrm{Mn}$ y $\mathrm{Co} / \mathrm{Zn}$ para las muestras analizadas presentan una variación entre 0.01 a 0.16 y 0.17 a 0.34 , respectivamente.

Los análisis microtermométricos sugieren dos poblaciones de inclusiones fluidas: una con una Th promedio de $136.5^{\circ} \mathrm{C}$ y una salinidad promedio de 4.2 por ciento en $\mathrm{NaCl}$ equivalente y otra con Th promedio de $136^{\circ} \mathrm{C}$ y una salinidad promedio de 11.4 por ciento en peso de $\mathrm{NaCl}$ equivalente.

Por su estructura, mineralogía, geoquímica elemental, fluidos y condiciones de depósito, la mineralización de Santa Rosa presenta una estrecha afinidad y relación genética con los depósitos de manantiales termales costeros actuales en la región. Por ello, su estudio permite establecer una correlación entre la metalogénesis de los depósitos de manganeso y los sistemas de manantiales hidrotermales someros activos.

Palabras clave: Hidrotermal, veta, brecha, romanechita, barita, ópalo, inclusiones fluidas, manantial termal, extensión tectónica.

\section{Abstract}

The Santa Rosa Mn-Ba deposit is located southwest of the town of San Nicolás and south of the Concepción Peninsula in Baja 
California Sur (Mexico). It consists of a manganese oxide vein-breccia striking NNW with a subvertical dip to the ENE that is exposed discontinuously over $500 \mathrm{~m}$. The deposit is hosted in conglomerates that belong to the Los Volcanes member of the Pliocene age San Nicolas Formation. The mineralization is characterized mainly by banded and brecciated structures with manganese oxides (romanèchite and pyrolusite), along with barite, opal, quartz, calcite, magnetite-maghemite and minor goethite.

The ore contains between 23.6 and $50.0 \mathrm{wt}$ \% $\mathrm{MnO}, 13.3$ and $32.4 \mathrm{wt} . \% \mathrm{SiO}$, and 9.9 and $13.7 \mathrm{wt}$. \% $\mathrm{BaO}$. In addition, the ores are significantly enriched in $\mathrm{Sr}, \mathrm{Sb}, \mathrm{As}, \mathrm{Cu}$ and $\mathrm{Zn}$. The NASC normalized REE profiles show a pronounced negative Eu anomaly and a more moderate negative Ce anomaly. The Fe/Mn and the Co/Zn ratios range between 0.01 and 0.16 , and 0.17 and 0.34 , respectively.

Microthermometry suggests two fluid inclusion populations: one with an average Th of $136.5{ }^{\circ} \mathrm{C}$ and estimated salinity of 4.2 wt. $\%$ equivalent $\mathrm{NaCl}$, and the other with an average Th of $136{ }^{\circ} \mathrm{C}$ and estimated salinity of $11.4 \mathrm{wt} . \%$ equivalent $\mathrm{NaCl}$.

The mineralogical and geochemical composition, together with the ore formation conditions and the geotectonic setting, suggest a genetic relation between the Santa Rosa deposit and the modern coastal hydrothermal systems of the Concepción Bay.

Keywords: Hydrothermal, vein, breccia, romanèchite, barite, opal, fluid inclusions, hot spring, extension tectonics.

\section{Introducción}

Los depósitos hidrotermales continentales de manganeso generalmente son depósitos pequeños y medianos, menores a las 50,000 Ton. de óxidos de Mn, emplazados en vetas (Laznicka, 1992). Un rasgo importante de los depósitos hidrotermales continentales es su afinidad y relación genética con los yacimientos epitermales y con los sistemas geotérmicos (Hewett, 1964; White, 1981; Crespo y Lunar, 1997; Miura y Hariya, 1997; Liakopoulos et al., 2001; Canet et al., 2005a).

Este tipo de depósitos de Mn es particularmente abundante en el noroeste de México y suroeste de Estados Unidos, donde forman vetas comúnmente encajonadas en rocas volcánicas ácidas a intermedias de edad terciaria (Mapes, 1956; González-Reyna, 1956; Hewett, 1964; Zantop, 1978; Roy, 1992, 1997). En ocasiones, estos depósitos ocurren como cuerpos lenticulares y concordantes y pueden asimismo estar encajonados en rocas sedimentarias de edades más recientes. Muchos de los depósitos conocidos más recientes se han formado en o cerca de la superficie terrestre, en un ambiente de manantiales termales (hot spring deposits). Por ello, pueden estar asociados a depósitos de sínter e incluso presentar estructuras estromatolíticas (Berger, 1985; Miura y Hariya, 1997; Canet et al., 2005a y b) y generalmente son de bajo potencial económico.

En la zona de Bahía Concepción, Baja California Sur, abundan las manifestaciones hidrotermales fósiles, como depósitos de óxidos de Mn emplazados en vetas, stockworks y brechas (El Gavilán, Mantitas, Minitas, Pilares, Trinidad y La Azteca), y actuales, como manantiales termales costeros formando precipitados en vetas y costras con óxidos de Mn (Rodríguez-Díaz, 2004; Camprubí et al., 2008). Los manantiales termales en la zona, Agua Caliente y Santispac, presentan depósitos que se componen principalmente de romanechita, pirolusita, todorokita, barita y ópalo (Canet et al., 2005b; Blanco-Florido, 2007). Las mineralizaciones en vetas están encajonadas normalmente en rocas volcánicas continentales del Oligoceno-Mioceno y están controladas por un sistema de fallas normales de dirección NO-SE, que tiene relación con eventos tectónicos extensivos terciarios asociados a la apertura del Golfo de California.

El depósito de Santa Rosa, localizado al sur de Península de Concepción, difiere significativamente de los otros depósitos en vetas por estar encajonado en conglomerados y sedimentos aluviales más recientes, del Plioceno Superior, y por presentar una mineralogía más simple (romanechita, barita y ópalo, esencialmente).

Por su mayor similitud en estructura y mineralización con respecto a las manifestaciones hidrotermales actuales, su estudio puede servir para establecer una correlación entre la metalogénesis de los depósitos de manganeso y los sistemas de manantiales hidrotermales activos.

\section{Ubicación y marco geológico}

Bahía Concepción se localiza en la costa oriental de la Península de Baja California Sur y tiene una orientación noroeste-sureste, presentando una forma alargada y semicerrada. Más al sur, entre ésta y la ciudad de Loreto, se encuentra otra bahía, de nombre San Nicolás, la cual se encuentra abierta al Golfo de California. Cerca de ella se ubica el depósito de Santa Rosa (26²8'37.94" N y $\left.111^{\circ} 33^{\prime} 6.4^{\prime \prime} \mathrm{W}\right)$, a unos $8 \mathrm{~km}$ al SO del poblado de San Nicolás (Figura 1). El acceso más directo a esta mina es a través de un camino sin asfaltar que parte de la Carretera Transpeninsular (Carretera Federal No. 1), aproximadamente a $97 \mathrm{~km}$ al norte de Loreto.

El basamento en la región de Bahía Concepción consiste en esquistos Paleozoicos que afloran muy escasamente $y$, mayoritariamente, en una serie de granitoides de edad cretácica, que en la zona de la Bahía de San Nicolás son de composición cuarzomonzonítica y granodiorítica (Figura 2; McFall, 1968; Casarrubias y Gómez-López, 1994; Ledesma-Vázquez, 2000). El Cenozoico está representado principalmente por la secuencia volcánica continental del Grupo Comondú del Oligoceno-Mioceno (McFall, 1968; Umhoefer et al., 2001; Figura 2). A su vez, rocas intrusivas 


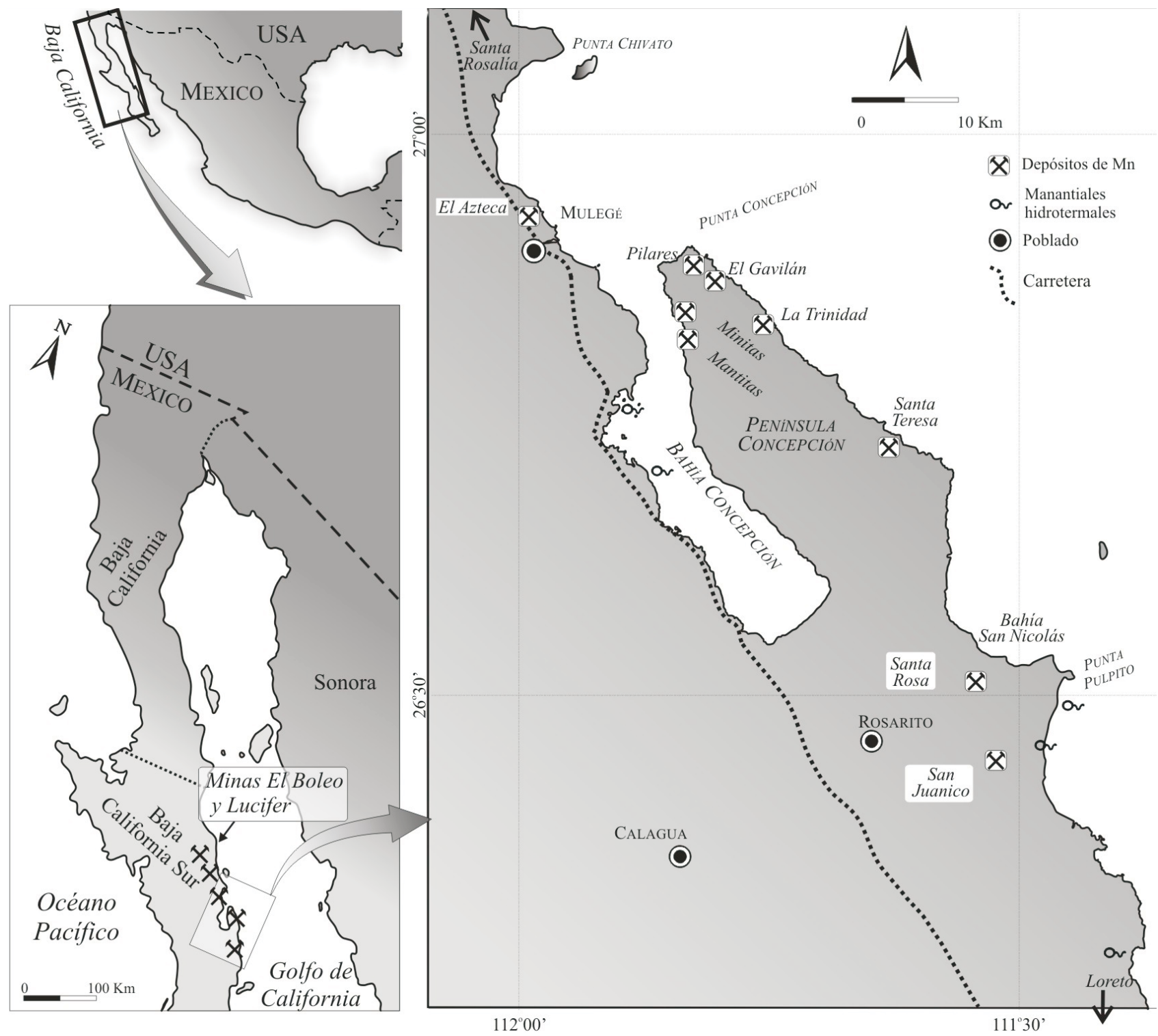

Figura 1. Localización del área de estudio, con las mineralizaciones de manganeso de Bahía Concepción, el depósito Santa Rosa y los manantiales termales costeros.

de composición gabroica y diorítica del Mioceno emplazadas en diques subverticales y sills cortan al basamento y al Grupo Comondú en la Península de Concepción (Noble, 1950; McFall, 1968).

Al sureste de Bahía Concepción (Figura 2), las formaciones Infierno y San Nicolás, de edad Plioceno Tardío, se encuentran sobreyaciendo discordantemente al Grupo Comondú (Ledesma-Vázquez, 2000; Ledesma-Vázquez et al., 1997, 2006). La Formación Infierno consiste de depósitos sedimentarios transgresivos, principalmente conglomerados volcánicos, areniscas, lodolitas, nódulos y capas de sílex con estructuras de reemplazamiento (Johnson et al., 1997; Rodríguez-Díaz, 2004). En el área de San Nicolás, sobreyaciendo a las rocas de la Formación El Salto del Grupo Comondú, se encuentra una serie de flujos de lava y tobas líticas, conglomerados, calizas y biocalcarenitas, a la Formación San Nicolás (Figura 3; Swalan y Smith, 1984; Ledesma-Vázquez et al., 2006). Esta formación se divide en cuatro miembros: $(a)$ la toba volcánica de San Antonio, (b) Los Volcanes, (c) Arroyo Amarillo y (c) El Saucito. El miembro Los Volcanes es un conglomerado matriz-soportado, depositado en ambiente de abanico aluvial. Dicho conglomerado contiene clastos de composición andesítica del Mioceno, con morfologías entre bien redondeadas y subangulosas. Es en este miembro donde se aloja la estructura mineralizada de Santa Rosa.

El Cuaternario consiste en depósitos vulcanosedimentarios y derrames lávicos adyacentes al área de Bahía San Nicolás y sedimentos aluviales, coluviales y marinos en toda la región. 


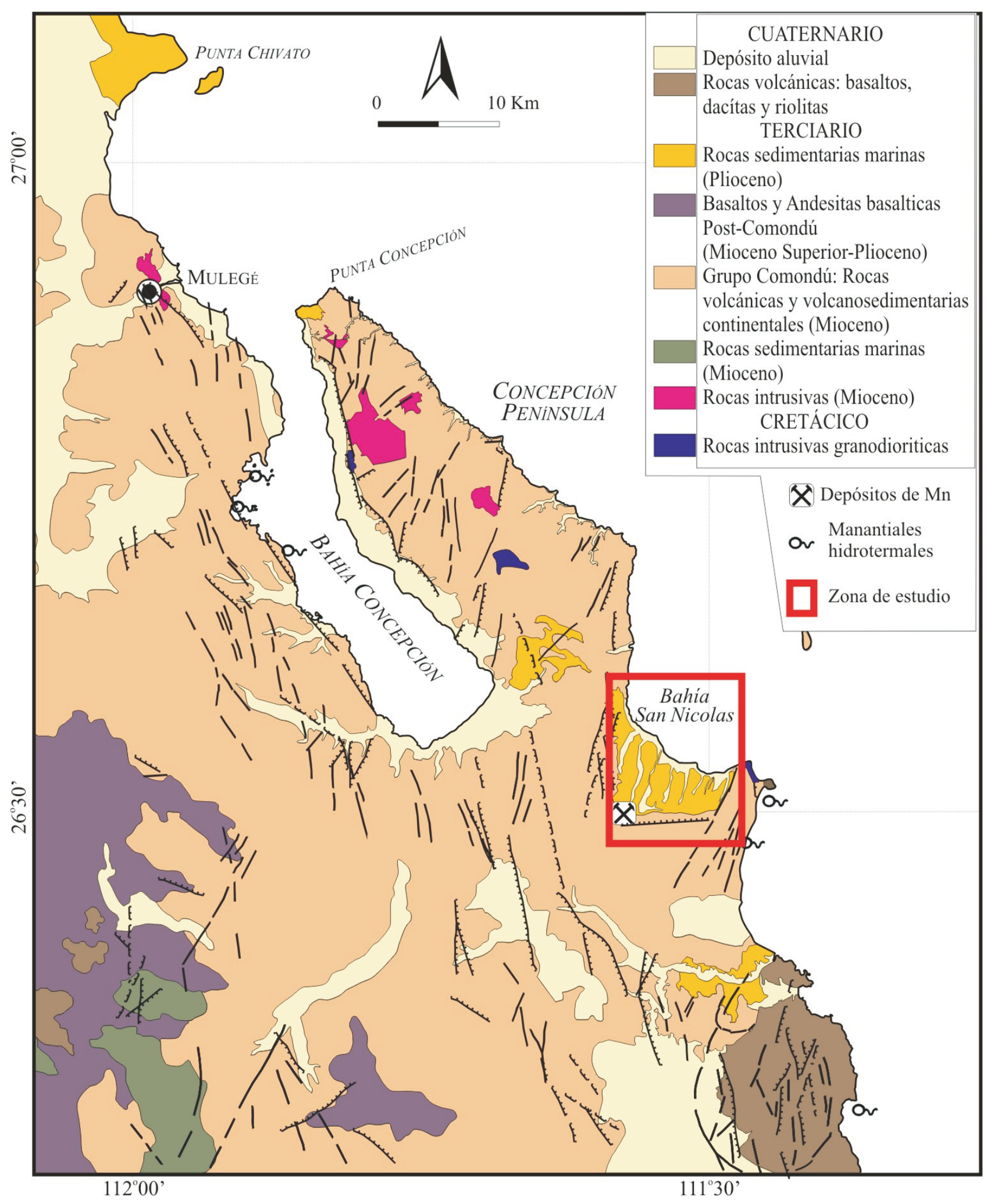

Figura 2. Mapa geológico general del área de Península Concepción y Bahía San Nicolás (Modificado de McFall, 1968; Maraver-Romero et al., 1997 y Ledesma-Vázquez et al., 2006). 


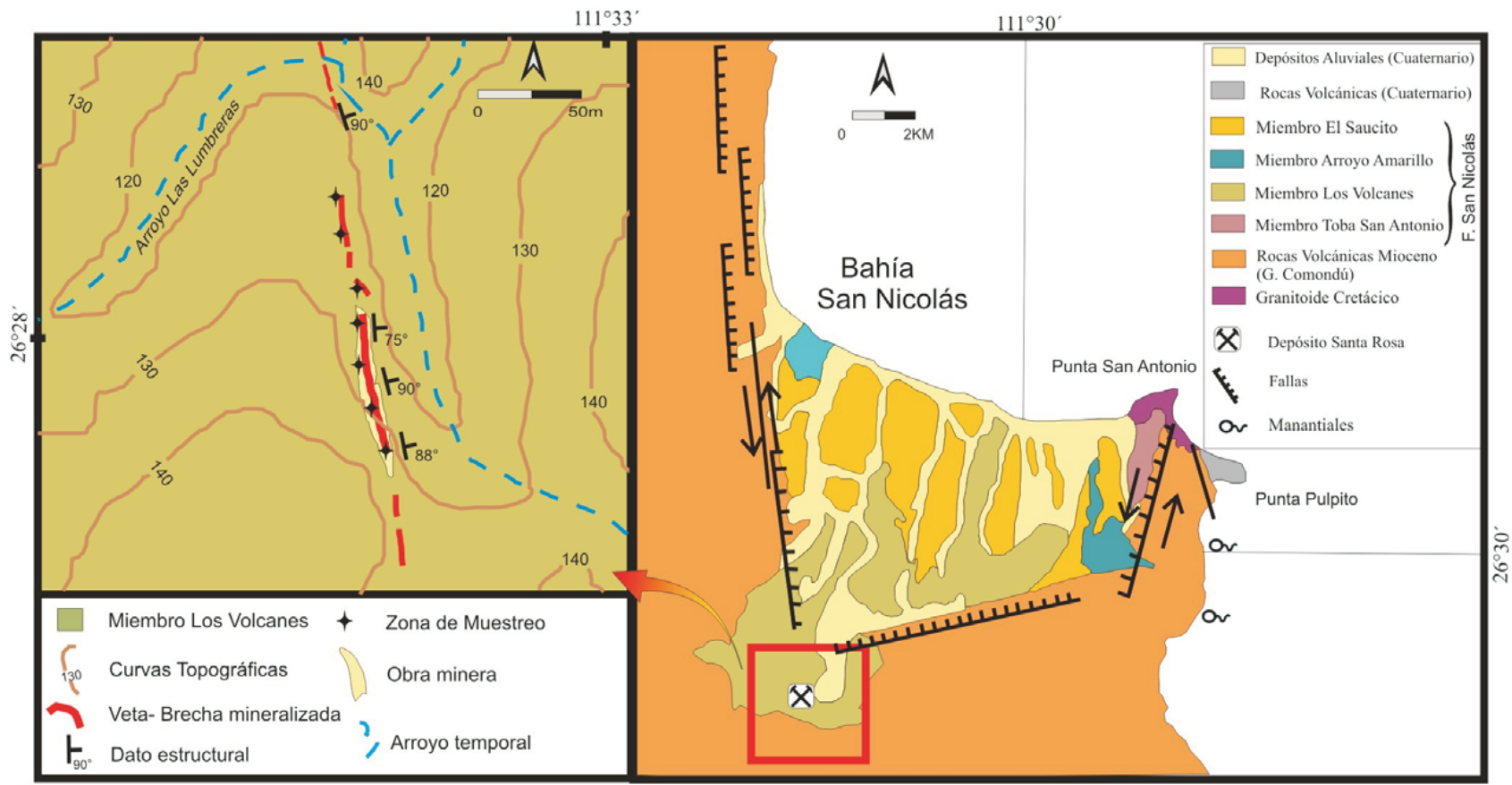

Figura 3. Mapa geológico del área de Bahía San Nicolás (Modificado de Ledesma-Vázquez et al., 2006) y del depósito Santa Rosa.

\section{Metodología}

La mineralogía fue determinada mediante observaciones macroscópicas, microscopía óptica de luz transmitida y reflejada, microscopía electrónica con análisis de energías dispersivas (SEM-EDS, por sus siglas en inglés), difracción de rayos $\mathrm{X}$ (DRX), y análisis de química mineral por microsonda electrónica (EMP, por sus siglas en inglés). Para la cuantificación geoquímica de las muestras mineralizadas se utilizó fluorescencia de rayos X (FRX) para elementos mayores y espectrometría de masas con plasma acoplado por inducción (ICP-MS, por sus siglas en inglés) para elementos menores y traza.

Para la caracterización mineralógica se empleó un microscopio óptico de luz transmitida y reflejada marca Carl Zeiss modelo Axiolab en el Instituto de Geofísica de la Universidad Nacional Autónoma de México (UNAM), acoplado a un software para microfotografías AxioVision 3.1. Para complementar las observaciones se ha empleado un equipo marca FEI Quanta 400, detector SSD de bajo vacío a una presión de 0.5 Tor y voltaje e $25 \mathrm{kV}$ y un software de análisis cualitativos EDAX Genesis del Centro Andaluz de Medio Ambiente (CEAMA), Granada, España. Con este equipo se han obtenido imágenes de electrones secundarios (SE, por sus siglas en inglés) y retrodispersados (BSE, por sus siglas en inglés) y análisis cualitativos de EDS.

El análisis mineralógico por la técnica de DRX se realizó en muestras separadas manualmente de mena y ganga, pulverizadas en mortero de ágata. Esta técnica permitió establecer con certeza la mineralogía del depósito, teniendo en cuenta que varias de las fases principales son de grano muy fino y/o poco cristalinas. Para los minerales de mena se utilizó un difractómetro de rayos X Philips PW1710 con monocromador de grafito, rendija automática, radiación $\mathrm{K}_{\alpha}$ del $\mathrm{Cu}(\lambda=1.545 \AA)$ y conexión on-line con una computadora; los datos fueron colectados cada 10 segundos en barridos continuos con paso angular de $0.02^{\circ} 2 \theta$, trabajando a $40 \mathrm{kV}$ y $40 \mathrm{~mA}$. El software empleado en la identificación fue $X$-powder con la base de datos PDF2.DAT del ICDD de la Facultad de Ciencias, Universidad de Granada (España). Para las fases de la ganga en la mineralización se usó un instrumento Panalytical X'Pert Diffractometer, con un ángulo de barrido de $4-100^{\circ} 2 \theta$, un paso de $0.017^{\circ} 2 \theta$ $\mathrm{y}$ un tiempo de adquisición de 50 segundos. La radiación utilizada fue $\mathrm{K} \alpha$ del $\mathrm{Cu}$ con una longitud $\lambda=1.54061 \AA$. Las condiciones de análisis fueron $40 \mathrm{kV}$ y $28 \mathrm{~mA}$. El software utilizado en la interpretación de los espectros de difracción fueron el DIFRAC/AT-(Siemens 1993)-EVA y el X'Pert High Score. Los análisis se llevaron a cabo en los Serveis Cientifico Tècnics de la Universitat de Barcelona (Cataluña, España).

La química mineral en las menas se determinó con un equipo de EMP CAMECA SX50, en los Serveis Cientifico Tècnics de la Universitat de Barcelona, dotado con 4 espectrómetros dispersivos en longitud de onda y un espectrómetro dispersivo en energía (PGT, USA). Los análisis se realizaron en distintos puntos de una lámina delgada pulida con mena de distinta textura (SR-c): bandeada y brechada. A partir de estos datos, se realizaron cálculos estequiométricos para obtener las fórmulas estructurales de los óxidos de Mn analizados y, consiguientemente, los 
contenidos elementales molares o en átomos por fórmula unidad (apfu) y agua de constitución o de coordinación. Las condiciones de análisis utilizadas para los minerales de manganeso fueron: aumento de 10.000, $20 \mathrm{ke} \mathrm{V,} 15 \mathrm{nA}$; se usaron como patrones: celestina $(\mathrm{Sr}, L \alpha)$, barita $(\mathrm{Ba}$, $L \alpha)$, corindón $(\mathrm{Al}, K \alpha)$, ortosa $(\mathrm{K}, K \alpha)$, albita $(\mathrm{Na}, K \alpha)$, hematites $(\mathrm{Fe}, K \alpha)$, rodonita $(\mathrm{Mn}, K \alpha)$, rutilo $(\mathrm{Ti}, K \alpha)$, cuarzo $(\mathrm{Si}, K \alpha)$, wollastonita $(\mathrm{Ca}, K \alpha)$, esfalerita $(\mathrm{Zn}, K \alpha)$ y periclasa $(\mathrm{Mg}, K \alpha)$.

Las inclusiones fluidas (IF) fueron estudiadas por la técnica de microtermometría en una platina térmica Linkam THMSG 600 del Centro de Geociencias de la UNAM, en Juriquilla, Querétaro. La reproducibilidad de las mediciones fue de $\pm 0.2^{\circ} \mathrm{C}$ por debajo de $\operatorname{los} 0^{\circ} \mathrm{C}$ (temperatura de fusión del hielo; Tfh) y de $\pm 2^{\circ} \mathrm{C}$ para las temperaturas de homogenización (Th). La salinidad se ha expresado en tanto por ciento en peso de cloruro de sodio equivalente ( $w t . \% \mathrm{NaCl}) . \mathrm{La}$ densidad del fluido y la salinidad fueron calculadas a partir de datos microtermométricos utilizando las ecuaciones de Bodnar (1993), Roedder (1984) y Zhang y Frantz (1987), asumiendo un sistema de $\mathrm{H}_{2} \mathrm{O}-\mathrm{NaCl}$ (Bodnar, 1983).

El análisis geoquímico de elementos mayores en la mineralización se realizó mediante FRX, usándose un espectrómetro de masas secuencial de FRX marca Siemens SRS 3000 del Laboratorio Universitario de Geoquímica Isotópica del Instituto de Geología de la UNAM. Los elementos traza fueron analizados en los laboratorios comerciales de Actlabs Laboratories (Ancaster, Ontario, Canadá) por medio de ICP-MS.

\section{Estructura del depósito Santa Rosa}

El depósito de Santa Rosa fue explotado durante los años sesenta por medio de unas trincheras de escaso desarrollo en las zonas más ricas en manganeso (MaraverRomero et al., 1997). Actualmente se pueden observar mejor las obras mineras a lo largo de un intervalo de $75 \mathrm{~m}$ de longitud, en la región más rica en mena de la estructura mineralizada.

La mineralización se encuentra sobre una ladera del arroyo Las Lumbreras y consiste en una veta-brecha de aproximadamente $500 \mathrm{~m}$ de longitud, expuesta en afloramientos discontinuos, con espesores variables desde $30 \mathrm{~cm}$ hasta $2 \mathrm{~m}$. En su mayor parte, el espesor de la estructura mineralizada es poco menos de $1 \mathrm{~m}$ y la mayor potencia ( $2 \mathrm{~m})$ se desarrolla únicamente en un intervalo de $52 \mathrm{~m}$ (Figura 3).

La veta-brecha tiene una disposición estructural de rumbo preferente NNO-SSE e inclinaciones hacia el ENE. Los datos de rumbos medidos se encuentran entre los $9^{\circ}$ y $50^{\circ} \mathrm{NO}$, preferentemente entre $\operatorname{los} 10^{\circ}$ y $20^{\circ}$, con buzamientos casi verticales, desde $90^{\circ}$ hasta $75^{\circ} \mathrm{NE}$.

La roca encajonante es un ortoconglomerado con una granulometría que varía desde gravas, de más de $1 \mathrm{~cm}$, hasta bloques, de hasta $30 \mathrm{~cm}$. Los clastos son en general subangulosos a subredondeados y mal clasificados y consisten en fragmentos de andesita y pórfido andesítico, con moderada oxidación por intemperismo.

La mineralización tiene principalmente estructuras bandeadas y brechadas, sin embargo, se observan también intervalos donde cementa a la roca detrítica encajonante (Figura 4). Las estructuras bandeadas se distinguen por la alternancia de bandas irregulares de coloración negroazulado y blanco-rosa, correspondientes a las zonas con predominio de óxidos de $\mathrm{Mn}$, y de ópalo y barita, respectivamente. Hacia los bordes y el centro de la estructura mineralizada predominan las bandas claras. Además, hay vetillas milimétricas que cortan a las bandas de óxidos y ganga. Dentro de las estructuras bandeadas se hallan lentes brechados decimétricos, que incluyen fragmentos de roca encajonante, mena y ganga, y presentan oquedades rellenas con óxidos de manganeso y ópalo formando texturas botroidales, así como fenocristales tabulares de barita de hasta $1 \mathrm{~cm}$ de longitud.

\section{Asociaciones minerales}

Los minerales de mena (óxidos de $\mathrm{Mn}$ ) consisten en romanechita, principalmente, y pirolusita, en cantidades subordinadas. Como minerales de ganga se encuentran barita, ópalo, cuarzo, calcita, magnetita-maghemita y goethita.

La romanechita $\left(\mathrm{Ba}, \mathrm{H}_{2} \mathrm{O}\right)_{2}\left(\mathrm{Mn}^{4+}, \mathrm{Mn}^{3+}\right)_{5} \mathrm{O}_{10}$ forma agregados botroidales de hasta $2 \mathrm{~cm}$ de diámetro, constituidos por cristales aciculares de coloración negro-azulada en muestra de mano. Además, se presenta en agregados radiales de cristales aciculares, especialmente cuando ocurre como relleno de cavidades y actúa como cementante en la brecha. En el microscopio óptico de luz reflejada se observa una zonación de cristales de romanechita que constituyen los botroides (Figura 5) y que geoquímicamente corresponde a un aumento progresivo y sutil en el contenido de $\mathrm{Ba}$, aunque es común encontrar alternancias de pulsos enriquecidos y empobrecidos en Ba (Figura 6).

La pirolusita $\mathrm{MnO}_{2}$ es escasa, con un contenido modal $\leq 5 \%$, desarrolla cristales de algunas decenas de $\mu \mathrm{m}$ de longitud que se disponen intersticialmente respecto a la romanechita, rellenando de forma pasiva micro-fracturas (vetillas) o la porosidad, o bien en pátinas recubriendo los clastos de la brecha. En ocasiones, los agregados de pirolusita son botroidales.

La barita $\mathrm{BaSO}_{4}$ ocurre como cristales tabulares de dimensiones variables, desde pocas micras hasta $1 \mathrm{~cm}$. Se asocia a los agregados de romanechita y desarrolla bandas porosas, rellena oquedades y actúa como cementante en la brecha. Observada en el SEM, usualmente muestra bordes corroídos y engolfados (Figura 6).

El ópalo $\mathrm{SiO}_{2} \cdot \mathrm{nH}_{2} \mathrm{O}$ se encuentra formando bandas coloformes de $1 \mathrm{~cm}$ de espesor, de textura homogénea, de color blanco, rosa y azul de forma intercalada; dichas bandas cubren superficialmente los óxidos de manganeso 


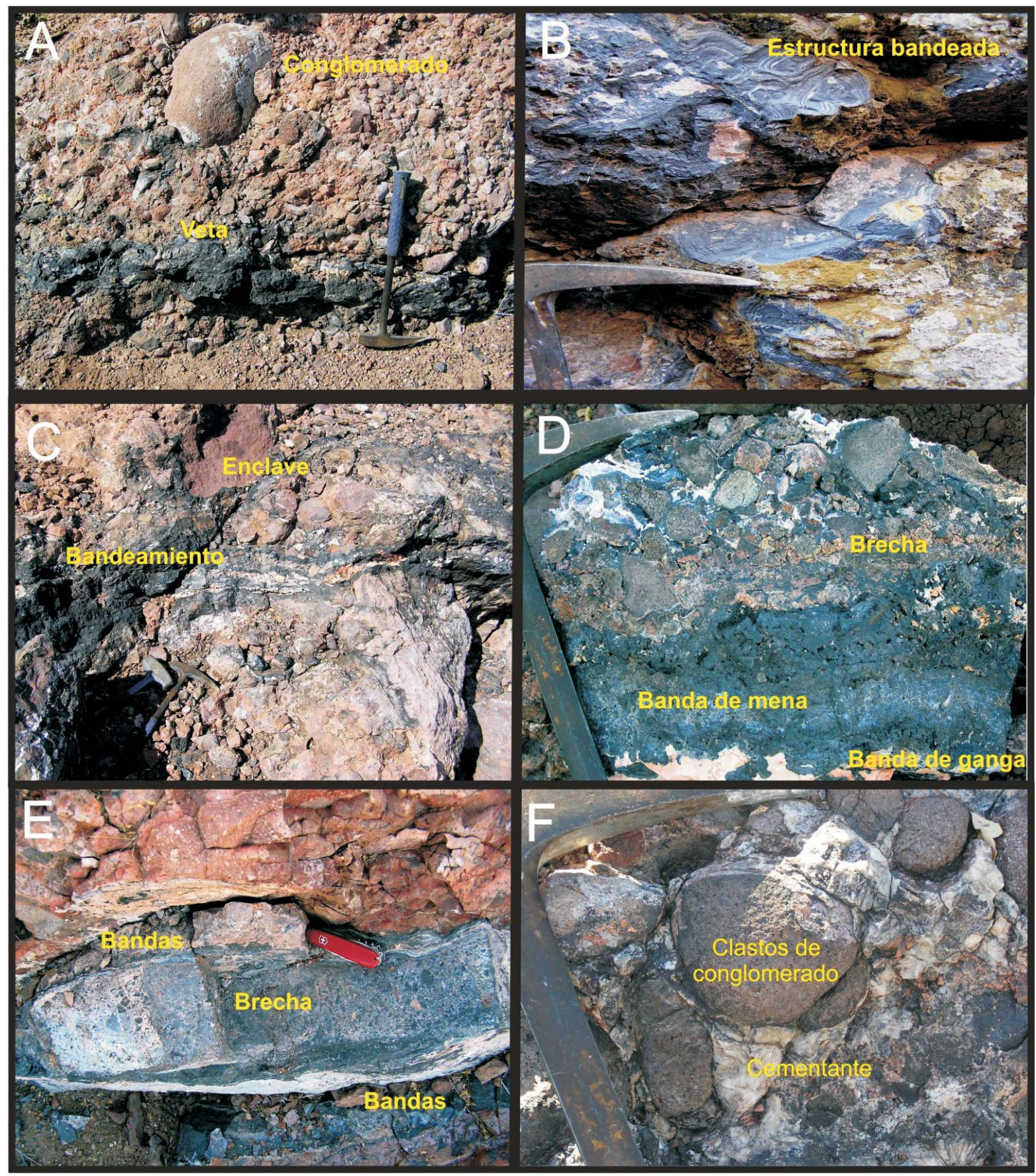

Figura 4. Fotografías del cuerpo mineralizado, la roca encajonante y sus detalles texturales. (A) Estructura tipo veta de óxidos de manganeso encajonada en conglomerados. (B) Textura bandeada coloforme de la veta con óxidos de manganeso. (C) Estructura veta-brecha con textura bandeada y bloques de roca encajonante alojados en la mineralización de Mn-Ba. (D) Aspectos texturales macroscópicos de la mineralización donde se muestran las bandas de óxidos de manganeso y ópalo, con brechamiento. (E) Estructura mineralizada con brecha en el centro y bandas de óxidos de manganeso hacia los lados. (F) Mineralización con ópalo y barita, cementando los conglomerados polimícticos.

y en algunas regiones se puede observar que el ópalo actúa también como cementante de la brecha (Figura 5).

El cuarzo aparece escasamente rellenado oquedades, alrededor de un $2 \%$ modal, como cristales pequeños de centenas de $\mu \mathrm{m}$.

La calcita se encuentra únicamente como relleno de porosidad, formando cristales euedrales de decenas de $\mu \mathrm{m}$.
La magnetita $\mathrm{Fe}_{3} \mathrm{O}_{4}$ se encuentra frecuentemente como fragmentos sueltos dentro de la brecha, de bordes subangulosos y tamaños de entre $0.5 \mathrm{~mm}$ a decenas de $\mu \mathrm{m}$. Frecuentemente, los fragmentos se encuentran fracturados y presentan bordes de alteración a maghemita. Estos cristales proceden de la roca encajonante. 

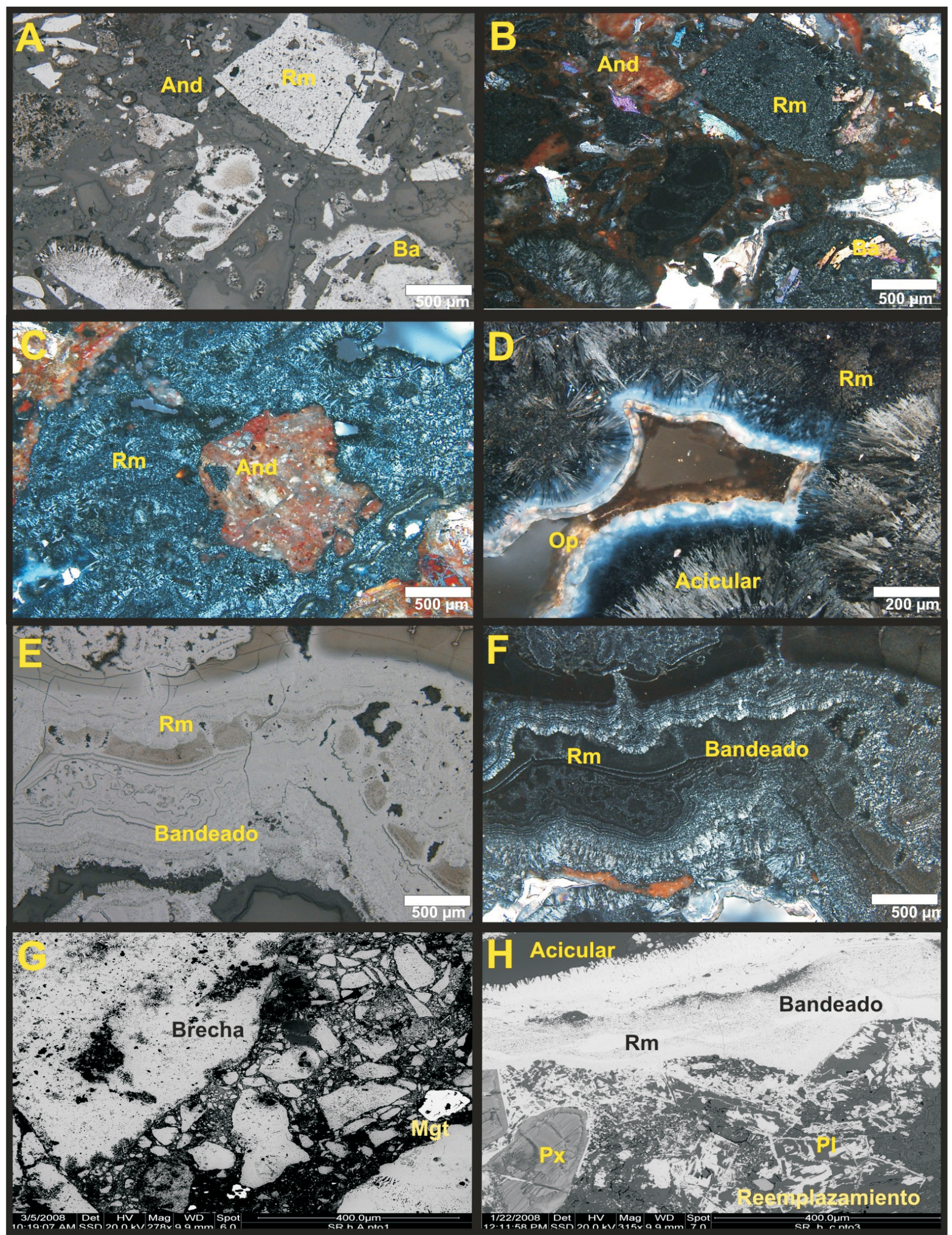

Figura 5. Texturas microscópicas de la mineralización de Mn-Ba. (A) Textura brechada con fragmentos de romanechita (Rm) masivos, bandeados coloformes y bandeado acicular. Algunos fragmentos alojan barita (Ba) tabular y fragmentos de andesita (And). Luz reflejada, sin analizador. (B) Luz reflejada, con analizador. (C) Textura brechada con romanechita con una marcada anisotropía y fragmentos de andesita parcialmente alterada a óxidos de Fe. Luz reflejada, con analizador. (D) Textura bandeada acicular de la romanechita con anisotropía y bandas de relleno de oquedades de ópalo (Op). Luz reflejada, con analizador. (E) Textura bandeada coloforme de romanechita con diferentes tonalidades de gris claro a beige. Luz reflejada, sin analizador. (F) Luz reflejada, con analizador. (G) Brecha de romanechita y fragmentos redondeados de magnetita-maghemita (Mgt). Imagen SEM-BSE. (H) Abajo, en la imagen, reemplazamientos de óxidos de manganeso en piroxeno ( $\mathrm{Px}$ ) y plagioclasa (Plg) de la roca encajonante; arriba, agregados bandeados de cristales microtabulares de romanechita. Imagen SEM-BSE 


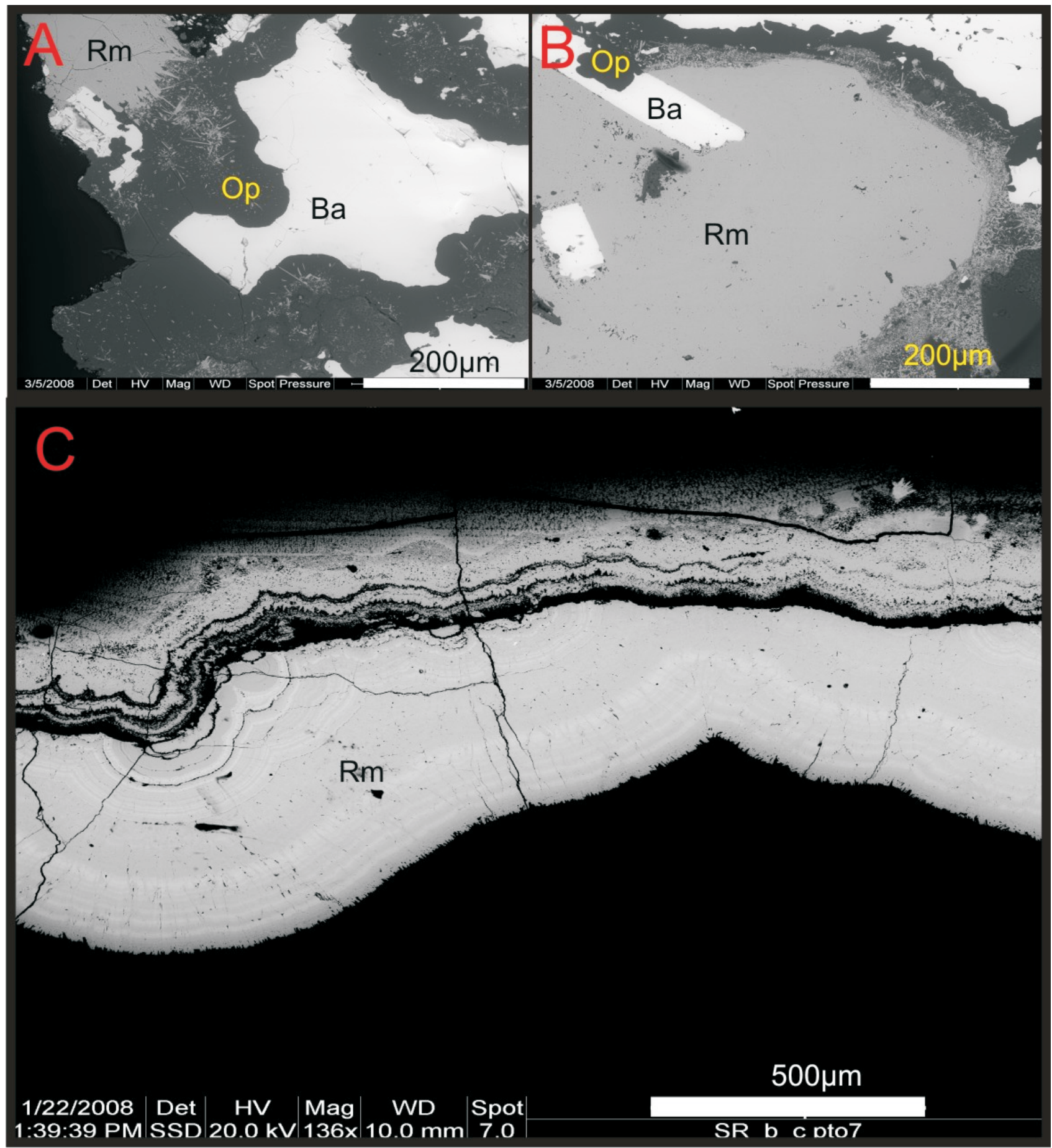

Figura 6. Imágenes de la mineralización de Mn-Ba obtenidas mediante microscopio SEM con electrones secundarios. (A) Bordes engolfados de cristales de barita (Ba), en blanco; reemplazamiento por ópalo (Op) en gris oscuro y romanechita $(\mathrm{Rm})$ masiva y acicular en gris claro. (B) Cristales de barita, en blanco, dentro de un agregado de romanechita, en gris claro, y ópalo reemplazando a la barita, en gris oscuro. (C) Agregados bandeados de romanechita de diferentes tonalidades causadas por variaciones de $\mathrm{Ba}$ en su contenido, bandas en gris claro ricas en $\mathrm{Ba}$.

La goethita $\alpha-\mathrm{FeO}(\mathrm{OH})$ se forma como alteración supergénica de los minerales de hierro de la brecha y forma pátinas, costras y, ocasionalmente, actúa como cementante entre pequeños fragmentos de roca y minerales en la brecha.
Pequeñas cantidades de minerales procedentes de la roca encajonante, como plagioclasa y clinopiroxeno, con tamaños desde decenas de $\mu \mathrm{m}$ hasta $0.5 \mathrm{~mm}$, y fragmentos de andesita milimétricos a centimétricos, muy alterados, se encuentran dentro de las vetas, cementados por la mineralización. 


\subsection{Difracción de Rayos X}

En los análisis de DRX realizados a tres muestras representativas de la mena (SR-a, SR-b y SR-c) es posible observar los picos característicos de la romanechita (2.41, 2.19 y $3.48 \AA$ ) (Smith y Mrose, 1974). De igual modo se determinó la presencia de cuarzo, plagioclasa y barita como fases accesorias (Figura 7).

Los análisis realizados en dos muestra representativas de la ganga (BC-8 y BC-10) presentaron como fase principal sílice amorfa (ópalo-C), caracterizado por tener una curvatura respecto a la línea base del difractograma; esta curvatura presenta su centro entre $20.8-21.75^{\circ} 2 \theta$, con un valor de FWHM de $0.2^{\circ}$ a $0.6^{\circ}$. Estas características son similares a las que Lynne et al. (2005), Herdianita et al. (2000) reportan para los depósitos de sílice amorfa en manantiales termales y sínters. En estos difractogramas se pueden observar otras fases minerales accesorias como cuarzo y barita (Figura 7).

\subsection{Química mineral}

La romanechita presenta una estructura cristalina semejante a la de la holandita $\mathrm{BaMn}_{8} \mathrm{O}_{16}$ y la todorokita (Mn, $\mathrm{Mg}, \mathrm{Ca}, \mathrm{Ba}, \mathrm{K}, \mathrm{Na})_{2} \mathrm{Mn}_{3} \mathrm{O}_{12} \cdot 3 \mathrm{H}_{2} \mathrm{O}$ (Bursill, 1979; Turner y Buseck, 1981; Vasconcelos, 1999). La estructura de la romanechita está construida por cadenas dobles y triples con octaedros de $\mathrm{MnO}_{6}$ con borde compartido que se une para formar grandes túneles con secciones cruzadas rectangulares, de dos por tres octaedros (Post, 1999). Los túneles son llenados con cationes de $\mathrm{Ba}^{2+}$ y moléculas de agua en una relación 1:2, además de pequeñas cantidades de otros cationes como $\mathrm{Na}^{+}, \mathrm{K}^{+}, \mathrm{Sr}^{2+}, \mathrm{Ca}^{2+}, \mathrm{Mg}^{2+}$ (Turner y Post, 1988; Vasconcelos, 1999); las cargas de los cationes del túnel están balanceados por substitución de Mn(III) por Mn(IV).

Los análisis de EMP fueron aplicados a romanechita con distinta textura (Tabla 1). Las romanechitas muestran fluctuaciones menores en su composición, con un rango de 76.30 a $69.66 \%$ en peso de $\mathrm{MnO}_{2}$ (total) y de 17.14 a 17.93 $\%$ en peso de $\mathrm{BaO}$. Los elementos menores son $\mathrm{Si}, \mathrm{Al}, \mathrm{Fe}$, $\mathrm{V}, \mathrm{Ca}, \mathrm{Mg}$ y $\mathrm{Cu}$. En general, los análisis químicos de la romanechita muestran una fórmula estructural: $\left(\mathrm{Ba}_{0.58-0.61}\right.$, $\left.\mathrm{H}_{2} \mathrm{O}_{1.37-1.40}\right)\left(\mathrm{Mn}^{4+}{ }_{4.53-4.56}, \mathrm{Fe}^{3+}{ }_{0.01-0.02}, \mathrm{Al}_{0.04-0.05}, \mathrm{Si}_{0.03}\right) \mathrm{O}_{10}$

La composición de la romanechita en los precipitados de los manantiales termales costeros de Agua Caliente (Tabla 1), a diferencia de la romanechita del depósito Santa Rosa, presenta un mayor contenido en $\mathrm{Si}, \mathrm{Mg}$, Ca y Fe (Tabla 1; Canet et al., 2005a). Sin embargo, las concentraciones de Ba en la romanechita de Santa Rosa son mayores que en Agua Caliente (Tabla 1).

\section{Secuencia paragenética}

La secuencia paragenética de la mineralización en el

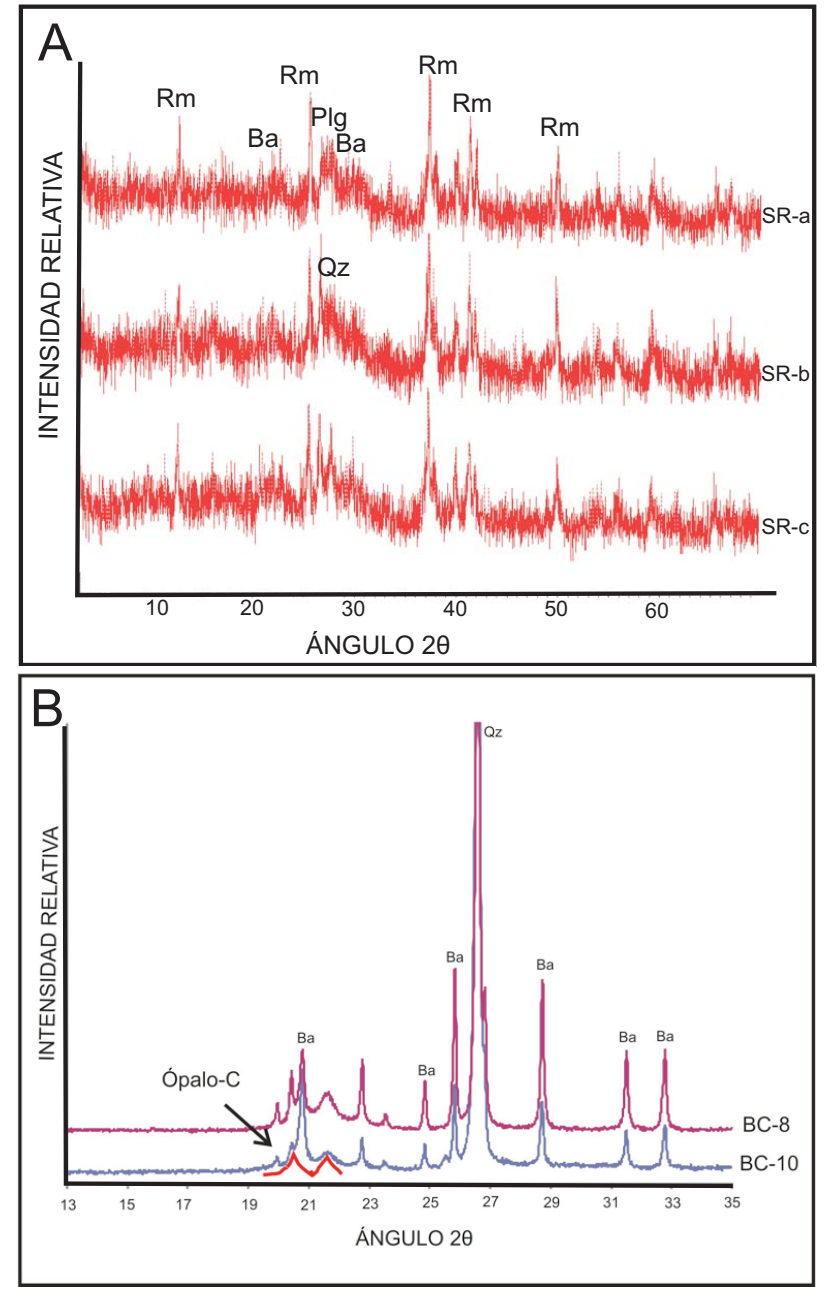

Figura 7. Espectros de difracción de rayos X. (A) Difractogramas de mena (SR-a, SR-b y SR-C). Fases identificadas: romanechita (Rm), barita (Ba), plagioclasa (Plg) y cuarzo (Qz). (B) Difractograma de ganga (BC-8 y BC-10): ópalo C, barita (Ba) y cuarzo (Qz).

depósito Santa Rosa pudo ser deducida por las observaciones en campo y en muestra de mano y por las relaciones texturales exhibidas en los microscopio petrográfico y electrónico (Figura 8).

Los granos de magnetita-maghemita contenidos hasta en un $3 \%$ modal en la veta-brecha mineralizada provienen de la roca encajonante. Ello queda evidenciado por sus similitudes texturales en los clastos de la roca encajonante, por el aislamiento de los fragmentos, sin relación con la mena y ganga, y por la morfología en los fragmentos, subangulosos a subredondeados, que indica un transporte.

El proceso hipogénico que dio lugar a la veta-brecha ocurrió mediante el desarrollo de dos etapas hidrotermales principales en la mineralización. Durante la primera etapa, veta-brecha y brecha, causante de la mineralización económica, se produjo la precipitación de barita y de romanechita. La romanechita registra variaciones en el contenido de bario, que en general aumentan hacia el final 
Tabla 1. Análisis mediante microsonda electrónica (EMP) de óxidos de Mn del depósito Santa Rosa (SR en este trabajo). Muestras de precipitados hidrotermales de manantiales Agua Caliente (AC, Canet et al., 2005a). Óxidos cuantificados en \% en peso. Óxidos no analizados (NM). Coeficientes de fórmula estequiométrica calculada (Coe). El agua se calculó con base en estequiometría.

\begin{tabular}{|c|c|c|c|c|c|c|c|c|c|c|c|c|c|c|c|c|c|c|c|c|}
\hline & $\mathrm{SiO}_{2}$ & $\mathrm{TiO}_{2}$ & $\mathrm{MnO}_{2}(\mathrm{t})$ & $\mathrm{1}_{2} \mathrm{O}_{3}$ & $\mathrm{~V}_{2} \mathrm{O}_{5}$ & $\mathrm{Fe}_{2} \mathrm{O}_{3}$ & $\operatorname{IgO}$ & $\mathrm{CaO}$ & $\mathrm{CuO}$ & $\mathrm{NiO}$ & $\mathrm{ZnO}$ & $\mathrm{CoO}$ & $\mathrm{SrO}$ & $\mathrm{BaO}$ & $\mathrm{PbO}$ & $\mathrm{Na}_{2} \mathrm{O}$ & $\mathrm{K}_{2} \mathrm{O}$ & $\mathrm{Sc}_{2} \mathrm{O}_{3}$ & $\mathrm{H}_{2} \mathrm{O}$ & Total \\
\hline & $\begin{array}{c}\% \\
\text { Peso }\end{array}$ & $\begin{array}{c}\% \\
\text { Peso }\end{array}$ & $\begin{array}{c}\% \\
\text { Peso }\end{array}$ & $\begin{array}{c}\% \\
\text { Peso }\end{array}$ & & $\begin{array}{c}\% \\
\text { Peso }\end{array}$ & & $\begin{array}{l}\% \\
\text { Peso }\end{array}$ & & $\begin{array}{c}\% \\
\text { Peso }\end{array}$ & $\begin{array}{c}\% \\
\text { Peso }\end{array}$ & $\begin{array}{c}\% \\
\text { Peso }\end{array}$ & $\begin{array}{c}\% \\
\text { Peso }\end{array}$ & $\begin{array}{c}\% \\
\text { Peso }\end{array}$ & $\begin{array}{c}\% \\
\text { Peso }\end{array}$ & $\begin{array}{c}\% \\
\text { Peso }\end{array}$ & $\begin{array}{c}\% \\
\text { Peso }\end{array}$ & $\begin{array}{c}\% \\
\text { Peso }\end{array}$ & $\begin{array}{c}\% \\
\text { Peso }\end{array}$ & $\begin{array}{c}\% \\
\text { Peso }\end{array}$ \\
\hline & 0.35 & 0 & 7560 & 0.51 & 0.19 & 0.11 & 0.04 & 0.21 & 0.08 & 0 & 0 & NM & 0 & 17.93 & 0.12 & 0 & 0 & NM & 0 & 95.22 \\
\hline $2-2$ & 0.33 & 0 & 75.81 & 43 & 0.16 & 0.11 & 0 & 0.2 & 0.04 & 0 & 0 & NM & 0 & 17.66 & 0.06 & 0 & 0 & $\mathrm{NM}$ & 0 & 94.8 \\
\hline D? & 0.32 & 0 & 76.33 & 0.43 & 0.2 & & 0.01 & 0.21 & 0.07 & 0 & 0 & NM & 0 & 17.14 & 0.19 & 0 & 0 & NIM & 0 & 90.00 \\
\hline 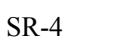 & 35 & 0 & 6.3 & 0.5 & 0.19 & 0.11 & 0 & 0.27 & 0.22 & 0 & 0 & NM & 0 & 17.89 & 0.11 & 0 & 0 & NM & 0 & 95.94 \\
\hline- & 0.39 & 0 & 76.52 & 0.45 & 0.21 & 0.25 & 0 & 0.23 & 0.15 & 0 & 0 & NM & 0 & 17.17 & 0.1 & 0 & 0 & $\mathrm{NIM}$ & 0 & 95.53 \\
\hline$C_{1}$ & 3.17 & 0 & 70.64 & 0.21 & 0 & 1.09 & 1.47 & 1.8 & NM & 0.01 & 0.16 & 0.1 & 0.19 & 9.91 & 0.09 & 0.52 & 0.35 & 0 & 8.6 & 98.32 \\
\hline $\mathrm{C}-2$ & 3.39 & 0 & 69.66 & 0.15 & 0 & 1.24 & 1.69 & 1.78 & NM & 0 & 0.11 & 0.04 & 0.2 & 10.07 & 0.02 & 0.48 & 0.32 & 0 & 8.55 & 97.69 \\
\hline
\end{tabular}

\begin{tabular}{|c|c|c|c|c|c|c|c|c|c|c|c|c|c|c|c|c|c|c|}
\hline \multirow{2}{*}{ Muestra } & $\mathrm{Si}$ & $\mathrm{Mn}^{4+}$ & $\mathrm{Al}$ & $\mathrm{V}$ & $\mathrm{Fe}^{3+}$ & $\mathrm{Mg}$ & $\mathrm{Ca}$ & $\mathrm{Cu}$ & $\mathrm{Ni}$ & $\mathrm{Zn}$ & $\mathrm{Sr}$ & $\mathrm{Ba}$ & $\mathrm{Pb}$ & $\mathrm{Na}$ & $\mathrm{K}$ & $\mathrm{H}_{2} \mathrm{O}$ & TOTAL & \multirow{2}{*}{ Total analítico } \\
\hline & $\mathrm{Coe}$ & $\mathrm{Coe}$ & Coe & Coe & Coe & $\mathrm{Coe}$ & Coe & $\mathrm{Coe}$ & $\mathrm{Coe}$ & $\mathrm{Coe}$ & Coe & $\mathrm{Coe}$ & $\mathrm{Coe}$ & Coe & Coe & $\mathrm{Coe}$ & $\mathrm{Coe}$ & \\
\hline SR-1 & 0.03 & 4.53 & 0.05 & 0.01 & 0.01 & 0.01 & 0.02 & 0.01 & 0 & 0 & 0 & 0.61 & 0 & 0 & 0 & 1.37 & 6.64 & 99.95 \\
\hline SR-2 & 0.03 & 4.56 & 0.04 & 0.01 & 0.01 & 0 & 0.02 & 0 & 0 & 0 & 0 & 0.6 & 0 & 0 & 0 & 1.38 & 6.65 & 99.54 \\
\hline SR-3 & 0.03 & 4.56 & 0.04 & 0.01 & 0.01 & 0 & 0.02 & 0 & 0 & 0 & 0 & 0.58 & 0 & 0 & 0 & 1.4 & 6.66 & 99.89 \\
\hline SR-4 & 0.03 & 4.53 & 0.05 & 0.01 & 0.01 & 0 & 0.02 & 0.01 & 0 & 0 & 0 & 0.6 & 0 & 0 & 0 & 1.37 & 6.65 & 100.71 \\
\hline SR-5 & 0.03 & 4.53 & 0.05 & 0.01 & 0.02 & 0 & 0.02 & 0.01 & 0 & 0 & 0 & 0.58 & 0 & 0 & 0 & 1.4 & 6.66 & 100.42 \\
\hline
\end{tabular}

de la etapa de mineralización, como se observó en estudios de SEM-EDS. Hacia el final de esta primera etapa, se formó pirolusita en cantidades accesorias. Esta generación de pirolusita hidrotermal se caracteriza por presentar cristales intercrecidos con los de romanechita. Durante una segunda etapa, también ligada al proceso hidrotermal, precipitó el ópalo, como relleno tardío de cavidades, con cantidades accesorias de barita, pirolusita y cuarzo.

Por último, como resultado de la alteración supergénica, se desarrollaron vetillas, costras y pátinas de calcita, goethita y de pirolusita (Etapa 3; Figura 8).

\section{Inclusiones fluidas}

Las mediciones microtermométricas fueron hechas en cuatro muestras de barita de la mineralización, con un to-

\begin{tabular}{|c|c|c|c|c|}
\hline \multirow{2}{*}{ Mineral } & \multirow{2}{*}{ Etapa 0} & \multirow{2}{*}{$\begin{array}{c}\text { Etapa } 1 \\
\text { Veta-Brecha }\end{array}$} & \multirow{2}{*}{ Etapa 2} & \multirow{2}{*}{ Etapa 3} \\
\hline & & & & \\
\hline \multicolumn{5}{|l|}{$\begin{array}{l}\text { Magnetita } \\
\text { Maghemita }\end{array}$} \\
\hline \multicolumn{5}{|c|}{ Romanechita } \\
\hline Barita & & $\cdots \rightarrow$ & $\cdots \cdots$ & \\
\hline Pirolusita & & & $\cdots \cdot$ & 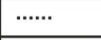 \\
\hline Ópalo & & & - & \\
\hline \multicolumn{5}{|l|}{ Cuarzo } \\
\hline Calcita & & & & $\cdots \cdots$ \\
\hline Goethita & & & & 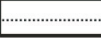 \\
\hline
\end{tabular}

Figura 8. Cuadro paragenético de la mineralización de Mn-Ba de Santa Rosa. tal de 49 inclusiones fluidas (IF) estudiadas; dos muestras corresponden a la etapa de formación de veta-brecha y dos muestras a la de brecha. La barita aparece como fenocristales tabulares intercrecidos con óxidos de manganeso. Fueron seleccionadas para este estudio las IF primarias y pseudosecundarias, excluyéndose todas aquellas que presentaron decrepitación o estrangulamiento. A temperatura ambiente, la mayoría de las IF presentes en las muestras son bifásicas (líquido + vapor), con grados de relleno ( $\mathrm{F}=$ $\mathrm{V}_{\text {Fase Liquido }} / \mathrm{V}_{\text {Total IF }}$ ) de $0.75-0.95$, y unifásicas (líquido). El tamaño de las IF varía en un rango de 5 a $80 \mu \mathrm{m}$. Las IF aisladas, agrupadas, dispersas y distribuidas al azar fueron consideradas como primarias o pseudosecundarias. Para proponer la veracidad y validación de los datos microtermométricos en las IF en barita, las cuales son susceptibles de sufrir procesos post-atrapamiento, se siguieron las recomendaciones de Ulrich y Bodnar (1988) y de Van den Kerkhof y Hein (2001).

La información derivada de la microtermometría se expone en la Tabla 2. Las mediciones microtermométricas registran temperaturas de homogeneización (Th) entre $108^{\circ}$ y $160{ }^{\circ} \mathrm{C}$, con un promedio de $126.47^{\circ} \mathrm{C}$. Las temperaturas de fusión del hielo (Tfh) medidas presentan un intervalo de $-1.8^{\circ}$ a $-8.6^{\circ} \mathrm{C}$. La salinidad calculada varía de 3.1 a 12.4 wt. $\% \mathrm{NaCl}$.

Los histogramas de Th y Tfh (Figura 9) y el gráfico de Th vs. salinidad (Figura 10) sugieren dos poblaciones de inclusiones, la primera con Th de $123^{\circ}$ hasta $156^{\circ} \mathrm{C}$, Tfh que varían de $-1.8^{\circ} \mathrm{a}-3.8^{\circ} \mathrm{C}$ y salinidades calculadas con valores entre 3.1 a $6.2 w t . \% \mathrm{NaCl}$ y una segunda población 


\begin{tabular}{|c|c|c|c|c|c|c|}
\hline Muestra & Mineralización & $\mathrm{n}$ & $\begin{array}{c}\mathrm{Th}\left({ }^{\circ} \mathrm{C}\right) \\
\mathrm{m} / \mathrm{p} / \mathrm{M}\end{array}$ & $\begin{array}{c}\mathrm{Tfh}\left({ }^{\circ} \mathrm{C}\right) \\
\mathrm{m} / \mathrm{p} / \mathrm{M}\end{array}$ & $\begin{array}{c}\text { Salinidad \% peso } \\
\mathrm{NaCl} \text { equivalente } \\
\mathrm{m} / \mathrm{p} / \mathrm{M}\end{array}$ & $\begin{array}{c}\text { Fases en } \\
\text { la IF }\end{array}$ \\
\hline SR-b & Brecha & 38 & $108 / 135.9 / 160$ & $-7.3 /-7.5 /-7.7$ & $10.9 / 11.1 / 11.3$ & $\mathrm{~L}+\mathrm{V}, \mathrm{L}$ \\
\hline SR-y & Brecha & 5 & 134 / $136 / 139$ & $-6.6 /-7.8 /-8.6$ & $10 / 11.5 / 12.4$ & $\mathrm{~L}+\mathrm{V}, \mathrm{L}$ \\
\hline SR-g & Veta-Brecha & 3 & $123 / 123.3 / 124$ & $-2.1 /-2.3 /-2.5$ & $3.6 / 3.9 / 4.2$ & $\mathrm{~L}+\mathrm{V}, \mathrm{L}$ \\
\hline SR-f & Veta-Brecha & 3 & $145 / 149.7 / 156$ & $-1.8 /-2.7 /-3.8$ & $3.1 / 4.4$ / 6.2 & $\mathrm{~L}+\mathrm{V}, \mathrm{L}$ \\
\hline
\end{tabular}

con Th moderadamente variables entre 108 y $160{ }^{\circ} \mathrm{C}$, Tfh con un intervalo entre $-6.6^{\circ} \mathrm{a}-8.6^{\circ} \mathrm{C}$ y salinidades en el rango de 10 a $12.4 w t . \% \mathrm{NaCl}$. Así, el primer grupo se caracteriza por Th de intervalo modal de $120^{\circ}-125^{\circ} \mathrm{C}$ y salinidad promedio de $4.2 w t . \% \mathrm{NaCl}$ y el segundo por Th de intervalo modal $110^{\circ}-115^{\circ} \mathrm{C}$ y salinidad promedio 11.4 wt. $\% \mathrm{NaCl}$.

\section{Geoquímica elemental}

La composición química de las menas de óxidos de manganeso de Santa Rosa y de tres muestras de precipitados del manantial termal de Agua Caliente (costras y veta de barita, ópalo, óxidos de manganeso y oxhidróxidos de hierro) se presentan en la Tabla 3. Los componentes químicos más abundantes en las muestras son: manganeso, de 23.6 a $50.0 \%$ en peso de $\mathrm{MnO}$, sílice, de 13.3 a $32.4 \%$ en peso de $\mathrm{SiO}_{2}$, y bario, de 9.9 a $13.7 \%$ en peso de $\mathrm{BaO}$. Además, en cantidades menores hay aluminio, con valores entre 1.8 y $9.5 \%$ en peso de $\mathrm{Al}_{2} \mathrm{O}_{3}$, hierro, de 0.5 a $4.2 \%$ en peso $\mathrm{Fe}_{2} \mathrm{O}_{3}$ (total), y calcio, de 0.2 a $2.6 \%$ en peso de $\mathrm{CaO}$. En cantidades por debajo del $1 w t . \%$, se detectan $\mathrm{Sr}$, $\mathrm{Sb}, \mathrm{As}, \mathrm{V}, \mathrm{W}, \mathrm{Cu}$ y Zn. Para las muestras de los manantiales termales, se observa la abundancia de sílice, de 31.0 a $46.2 \%$ en peso de $\mathrm{SiO}_{2}$; bario, de 18.1 a $30.0 \%$ en peso de $\mathrm{BaO}$; aluminio, con valores entre 1.8 y $5.0, \%$ en peso de $\mathrm{Al}_{2} \mathrm{O}_{3}$; hierro, de 0.93 a $2.61 \%$ en peso $\mathrm{Fe}_{2} \mathrm{O}_{3}$; y con valores por debajo del $1 \%$ en peso, se detectaron $\mathrm{Mn}, \mathrm{Ca}$, $\mathrm{Sr}, \mathrm{Sb}, \mathrm{V}$ y As.

La concentración total de los elementos de tierras raras (REE, por sus siglas en inglés) de las menas en Santa Rosa varía de 18.9 a $66.6 \mathrm{ppm}$ y en los precipitados hidrotermales de Agua Caliente de 8.7 a 15.8 ppm. En particular, las concentraciones de los elementos ligeros de la tierras raras, La-Eu, (LREE, por sus siglas en inglés) en las menas de Santa Rosa varían en un rango de 17.6 a 60.7 ppm, mientras que los elementos de tierras raras pesadas, Gd-Lu, (HREE, por sus siglas en inglés) oscilan alrededor de los 5.9 ppm en las muestras de Santa Rosa, lo que denota una mayor concentración de LREE que de HREE. Los LREE en los precipitados en Agua Caliente varían entre 7.1 y $14.2 \mathrm{ppm}$.

El contenido de REE normalizado al compuesto de lutita de América del Norte (NASC, por sus siglas en in- glés; Taylor y McLennan, 1985) se muestra en la Figura 11. Los perfiles normalizados de las REE de las menas de Santa Rosa muestran una anomalía negativa de Eu y una moderada anomalía negativa de Ce. En los precipitados del manantial termal Agua Caliente se observa solamente una anomalía negativa en Ce poco pronunciada, lo cual coincide con las muestras de mena de Santa Rosa. Las anomalías negativas de $\mathrm{Eu} / \mathrm{Eu}^{*}$ y $\mathrm{Ce} / \mathrm{Ce}^{*}$, calculadas mediante la expresión de McLennan (1989), para las muestras de Santa Rosa varían en un rango de 0.017 a 0.030 y 0.074 a 0.123 , respectivamente. Para los precipitados de Agua Caliente los valores de $\mathrm{Ce} / \mathrm{Ce}^{*}$ se sitúan entre 0.053 y 0.112

Las relaciones de $\mathrm{Fe} / \mathrm{Mn}$ varían entre 0.010 y 0.160 y las de $\mathrm{Co} / \mathrm{Zn}$ entre 0.173 y 0.338 para las muestras de la mena de Santa Rosa. En las muestras de precipitados del Agua Caliente, $\mathrm{Fe} / \mathrm{Mn}$ varía de 2.709 a 16.106 y Co/Zn de 0.029 a 0.267 .

\section{Discusión}

\subsection{Contexto tectónico}

La existencia de manifestaciones hidrotermales actuales y fósiles en el área de Bahía Concepción y Bahía San Nicolás presenta una gran relevancia por la estrecha relación que muestran entre sí y porque ponen en evidencia los procesos hidrotermales y metalogenéticos propios de un contexto volcánico y tectónico extensivo. El estudio mineralógico y geoquímico, y la caracterización de los fluidos mineralizantes pueden permitir establecer una correlación metalogenética de los depósitos de manganeso y los sistemas de manantiales hidrotermales someros activos.

Las rocas predominantes que alojan a la mayoría de las manifestaciones hidrotermales fósiles y actuales son rocas volcánicas continentales de composición intermedia y de edad Oligoceno-Mioceno del Grupo Comondú, relacionadas con un contexto de tipo arco (Hausback, 1984; Bigioggero et al., 1995; Ledesma-Vázquez y Johnson, 2001; Johnson y Ledesma-Vázquez, 2001; Umhoefer et al., 2001). Durante el Mioceno Medio y Superior el área se vio sujeta a eventos tectónicos extensivos relacionados con la apertura del Golfo de California, desarrollándose cuencas limitadas por fallas normales de orientación preferente NO-SE. Tales fallas han servido como conductos 

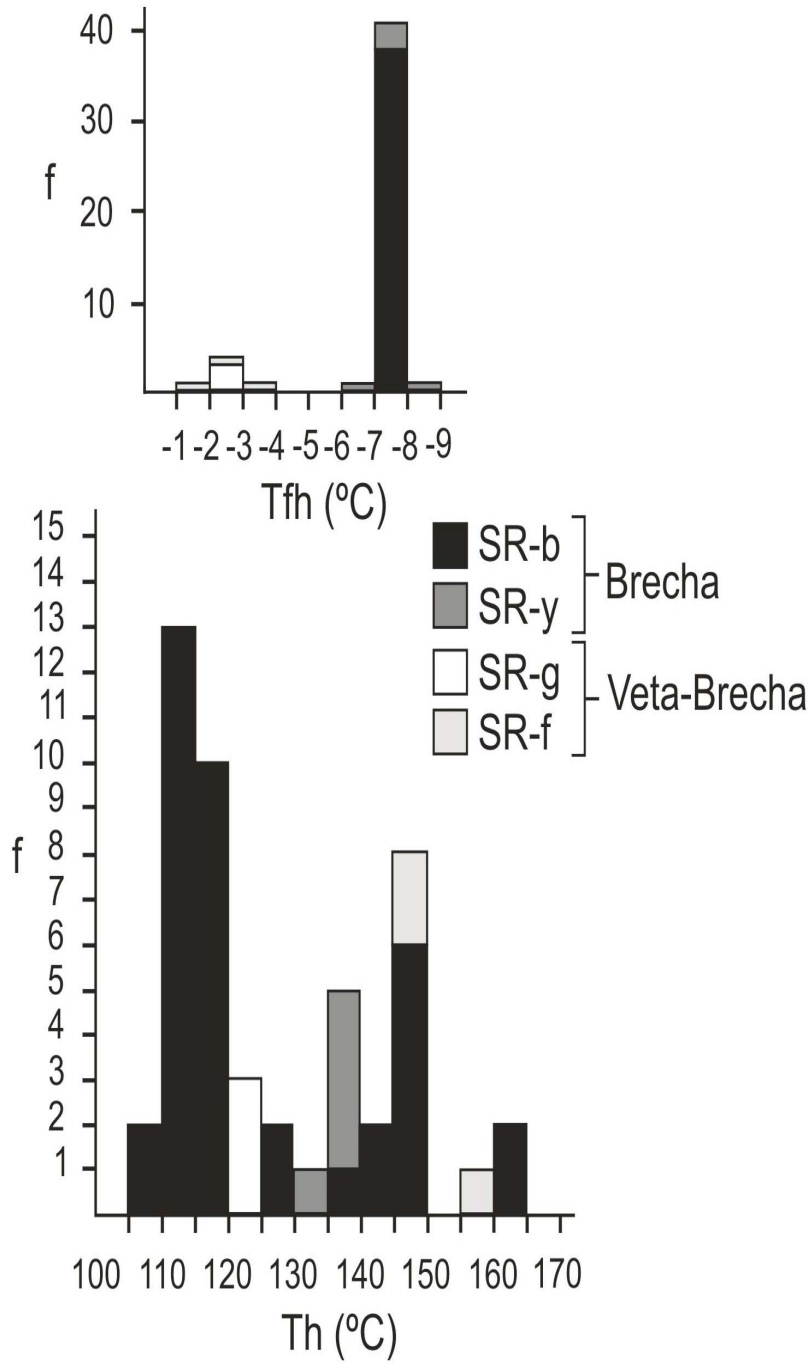

Figura 9. Histogramas mostrando la distribución de la temperatura de fusión del hielo $\left(\mathrm{Tfh}^{\circ} \mathrm{C}\right.$ ) y temperatura de homogenización $\left(\mathrm{Th}{ }^{\circ} \mathrm{C}\right)$, para inclusiones analizadas en cristales de barita de Santa Rosa, de las etapas de mineralización de veta-brecha y brecha.

para mineralizaciones hidrotermales de manganeso en las siguientes áreas: El Gavilán, Santa Teresa, Trinidad, Mantitas, Minitas y La Azteca en Bahía Concepción y El Boleo y Lucifer en la Cuenca de Santa Rosalía. Además, canalizan actualmente la circulación de fluidos hidrotermales de los manantiales termales costeros (Freiberg, 1983; Casarrubias y Gómez-López, 1994; Prol-Ledesma et al., 2004; Rodríguez-Díaz, 2004; Canet et al., 2005a; Camprubí et al., 2008; Del Rio-Salas et al., 2008). En la región entre Bahía San Nicolás y Loreto existe un sistema de fallas normales y transtensionales orientadas al NO-SE y ENE-OSO, respectivamente, de edad Mioceno Superior, algunas de las cuales se reactivaron en el Plioceno (Umhoefer et al., 2002). Un posible mecanismo capaz de promover la migración y precipitación de fluidos en Santa Rosa fue la reactivación del sistema de fallas Bahía San Nicolás y Loreto. La reapertura de las fallas se traduce en

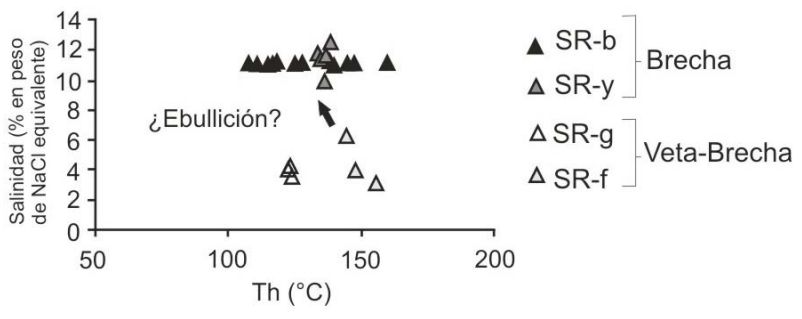

Figura 10. Gráfico de temperatura de homogenización (Th) vs. salinidad (\% en peso de $\mathrm{NaCl}$ equivalente), para inclusiones analizadas en cristales de barita de la veta-brecha y brecha del depósito Santa Rosa. Se observa una posible ebullición donde se aumenta drásticamente la salinidad de los fluidos debido al escape de vapor y su temperatura disminuye debido a la descompresión.

una reducción sustancial y repentina en la presión local del fluido en ciertos sitios, produciendo una migración de fluidos y la subsiguiente precipitación de minerales debido a procesos de ebullición y mezcla. Esta suposición puede apoyarse en la similitud de estructuras de vetas-brechas de óxido de manganeso de orientación NO-SE y ENE-OSO de la mineralización del Plioceno de San Juanico, ubicada al sur del depósito de Santa Rosa (González-Reyna, 1956; Terán-Ortega y Ávalos-Zermeño, 1993).

\subsection{Asociaciones minerales y Paragénesis mineral}

Alrededor de los manantiales termales de Agua Caliente existen precipitados en forma de vetas y costras de todorokita, romanechita, oxhidróxidos de $\mathrm{Fe}$, pirita, cinabrio, barita, calcita y ópalo A y A/CT (Canet et al., 2005a, 2005b; Blanco-Florido, 2007). Esta asociación mineralógica es similar a la de Santa Rosa, donde predominan la romanechita, barita, calcita y ópalo C. La asociación mineral de Santa Rosa es asimismo similar a la observada en otros depósitos de Mn del área de Bahía Concepción (El Gavilán, Mantitas, Minitas, Santa Teresa, Trinidad, La Azteca y San Juanico; González-Reyna, 1956; RodríguezDíaz, 2004; Camprubí et al., 2008) y Boleo y Lucifer en la Cuenca de Santa Rosalía (Freiberg, 1983; Del Río-Salas et al., 2008). Las asociaciones minerales encontradas en Santa Rosa y Agua Caliente coinciden con las de depósitos de ambiente manantial termal (hot spring) como en Hokkaido, Japón (Miura y Hariya, 1997); Artillery Mountains, Arizona (Mouat, 1962); Golconda, Nevada (Kerr, 1940), $\mathrm{y}$ algunos epitermales con relación con manantiales termales como Vani, Milos, Grecia (Liakopoulos et al., 2001) y Córdoba, Argentina (Leal, 2002).

La mena principal en Santa Rosa, romanechita, presenta una química mineral con valores altos de $\mathrm{BaO}$ que oscilan entre 17 y $18 \%$ en peso, moderados de $\mathrm{Si}, \mathrm{Al}$ y $\mathrm{V}$ y pobres (por debajo de $1 \%$ en peso) de $\mathrm{Ca}, \mathrm{Mg}, \mathrm{Fe}$ y $\mathrm{Cu}$. Debido a su estructura interna la romanechita puede tener cantidades variables de $\mathrm{Ba} \mathrm{y}_{2} \mathrm{O}$, por su relación de reemplazamiento isomórfico, en proporciones generalmente 1:2, respectivamente (Wadsley, 1953; Bursill, 1979; 
Tabla 3. Composición química de muestras de mena del depósito Santa Rosa (SR) y depósitos del manantial Agua Caliente (Map). Los elementos mayores están en \% en peso y los elementos traza en partes por millón (ppm), excepto el Au en partes por billón (ppb). Cuantificación de la pérdida por calcinación (PXC).

\begin{tabular}{|c|c|c|c|c|c|c|c|c|c|c|c|c|}
\hline \multirow{2}{*}{ Muestras } & $\mathrm{SiO}_{2}$ & $\mathrm{TiO}_{2}$ & $\mathrm{Al}_{2} \mathrm{O}_{3}$ & $\mathrm{Fe}_{2} \mathrm{O}_{3 \text { Total }}$ & $\mathrm{MnO}$ & $\mathrm{MgO}$ & $\mathrm{CaO}$ & $\mathrm{Na}_{2} \mathrm{O}$ & $\mathrm{K}_{2} \mathrm{O}$ & $\mathrm{P}_{2} \mathrm{O}_{5}$ & \multirow{2}{*}{$\mathrm{PXC}$} & \multirow{2}{*}{ Total } \\
\hline & $\%$ Peso & $\%$ Peso & $\%$ Peso & $\%$ Peso & $\%$ Peso & $\%$ Peso & $\%$ Peso & $\%$ Peso & $\%$ Peso & $\%$ Peso & & \\
\hline SR-b & 32.43 & 0.55 & 9.46 & 4.18 & 23.58 & 0.15 & 2.62 & 1.13 & 1.21 & 0.03 & 6.46 & 97.1 \\
\hline SR-c & 13.32 & 0.09 & 1.91 & 0.54 & 49.98 & 0 & 0.45 & 0 & 0.09 & 0 & 9.3 & 96.22 \\
\hline SR-h & 18.09 & 0.09 & 1.8 & 0.68 & 42.81 & 0 & 0.25 & 0 & 0.24 & 0 & 8.11 & 96 \\
\hline SR-Rod & 18.67 & 0.18 & 3.21 & 1.14 & 44.85 & 0.01 & 0.52 & 0 & 0.67 & 0 & 8.73 & 96.93 \\
\hline Map-5B & 46.18 & 0.24 & 5.02 & 2.14 & 0.12 & 0 & 0.93 & 0.48 & 0.7 & 0 & 20.85 & 76.66 \\
\hline Map-7 & 41.39 & 0.1 & 1.83 & 2.61 & 0.87 & 0 & 0.42 & 0 & 0 & 0 & 20.05 & 67.27 \\
\hline Map-10 & 30.96 & 0.19 & 4.28 & 0.93 & 0.25 & 0 & 1.18 & 0.73 & 0.31 & 0 & 24.81 & 63.64 \\
\hline
\end{tabular}

\begin{tabular}{|c|c|c|c|c|c|c|c|c|c|c|c|}
\hline \multirow{2}{*}{ Muestras } & $\mathrm{V}$ & $\mathrm{Cr}$ & $\mathrm{Co}$ & $\mathrm{Ni}$ & $\mathrm{Cu}$ & $\mathrm{Zn}$ & Ga & $\mathrm{Ge}$ & As & $\mathrm{Rb}$ & $\mathrm{Sr}$ \\
\hline & ppm & ppm & ppm & ppm & ppm & ppm & ppm & ppm & ppm & ppm & ppm \\
\hline SR-b & 778 & 50 & 55 & 40 & 620 & 280 & 48 & 15 & 1380 & 45 & 1020 \\
\hline SR-c & 1410 & 20 & 77 & 30 & 1140 & 340 & 60 & 28 & 1340 & 11 & 796 \\
\hline SR-h & 1310 & 50 & 57 & 20 & 1330 & 330 & 52 & 20 & 937 & 14 & 898 \\
\hline SR-Rod & 23 & 20 & 27 & 20 & 30 & 80 & 35 & $<1$ & 30 & 61 & 1020 \\
\hline Map-5B & 50 & 90 & 13 & $<20$ & 20 & 230 & 25 & 2 & 56 & 25 & 2980 \\
\hline Map-7 & 67 & $<20$ & 24 & $<20$ & 30 & 260 & 17 & 6 & 59 & 3 & 4740 \\
\hline Map-10 & 105 & 20 & 9 & $<20$ & 80 & 310 & 18 & 2 & 102 & 14 & 6990 \\
\hline \multirow{2}{*}{ Muestras } & Y & $\mathrm{Zr}$ & $\mathrm{Nb}$ & Mo & $\mathrm{Ag}$ & $\mathrm{Au}$ & In & $\mathrm{Sn}$ & $\mathrm{Sb}$ & Cs & $\mathrm{Ba}$ \\
\hline & ppm & ppm & ppm & ppm & ppm & $\mathrm{ppb}$ & ppm & ppm & ppm & ppm & ppm \\
\hline SR-b & 8 & 51 & 2 & 98 & 0.8 & 5 & $<0.2$ & $<1$ & 569 & 18.6 & 99000 \\
\hline SR-c & 4 & 6 & $<1$ & 128 & 0.5 & 5 & $<0.2$ & $<1$ & 980 & 6.2 & 124000 \\
\hline SR-h & 4 & 5 & $<1$ & 88 & 0.5 & 5 & $<0.2$ & $<1$ & 457 & 4 & 137000 \\
\hline SR-Rod & 4 & 26 & $<1$ & 2 & 0.5 & 8 & $<0.2$ & $<1$ & 30.6 & 42.6 & 65700 \\
\hline Map-5B & 4 & 29 & $<1$ & 5 & $<0.5$ & 5 & $<0.2$ & $<1$ & 93.6 & 17 & 181000 \\
\hline Map-7 & 4 & $<5$ & $<1$ & $<2$ & $<0.5$ & 10 & $<0.2$ & $<1$ & 89 & 2.2 & 260000 \\
\hline Map-10 & 5 & 22 & $<1$ & $<2$ & $<0.5$ & 8 & $<0.2$ & 7 & 89.1 & 10.2 & 300000 \\
\hline \multirow{2}{*}{ Muestras } & $\mathrm{La}$ & $\mathrm{Ce}$ & $\operatorname{Pr}$ & $\mathrm{Nd}$ & $\mathrm{Sm}$ & $\mathrm{Eu}$ & $\mathrm{Gd}$ & $\mathrm{Tb}$ & Dy & Ho & Er \\
\hline & ppm & ppm & ppm & ppm & ppm & ppm & ppm & ppm & ppm & ppm & ppm \\
\hline SR-b & 14.6 & 30.4 & 3.04 & 10.6 & 2 & 0.04 & 1.8 & 0.3 & 1.7 & 0.3 & 0.8 \\
\hline SR-c & 7.1 & 5.8 & 1.13 & 3.3 & 0.6 & 0.04 & 0.7 & $<0.1$ & 0.5 & $<0.1$ & 0.3 \\
\hline SR-h & 6.2 & 7.3 & 1.02 & 2.7 & 0.4 & 0.04 & 0.4 & $<0.1$ & 0.4 & $<0.1$ & 0.2 \\
\hline SR-Rod & 3.6 & 8.7 & 1 & 3.5 & 0.8 & 0.03 & 0.7 & 0.1 & 0.7 & 0.1 & 0.3 \\
\hline Map-5B & 4.4 & 7.2 & 0.78 & 1.6 & 0.2 & 0.06 & 0.3 & 0.3 & 0.4 & $<0.1$ & 0.3 \\
\hline Map-7 & 1.5 & 2.7 & 0.54 & 2.2 & 0.1 & 0.04 & 0.5 & 0.1 & 0.4 & 0.1 & 0.3 \\
\hline Map-10 & 3.9 & 5.9 & 0.62 & 1.3 & 0.1 & 0.05 & 0.6 & $<0.1$ & 0.4 & 0.2 & 0.3 \\
\hline \multirow{2}{*}{ Muestras } & $\mathrm{Tm}$ & $\mathrm{Yb}$ & $\mathrm{Lu}$ & Hf & $\mathrm{Ta}$ & W & $\mathrm{Tl}$ & $\mathrm{Pb}$ & $\mathrm{Bi}$ & Th & $\mathrm{U}$ \\
\hline & ppm & ppm & ppm & ppm & ppm & ppm & ppm & ppm & ppm & ppm & ppm \\
\hline SR-b & 0.05 & 0.8 & 0.13 & 0.7 & $<0.1$ & 1730 & 6.6 & 245 & 0.6 & 0.7 & 1.2 \\
\hline SR-c & $<0.05$ & 0.3 & 0.05 & $<0.2$ & $<0.1$ & 2600 & 7.5 & 49 & $<0.4$ & $<0.1$ & 3 \\
\hline SR-h & $<0.05$ & 0.2 & 0.04 & $<0.2$ & $<0.1$ & 2380 & 6.7 & 82 & $<0.4$ & $<0.1$ & 2.7 \\
\hline SR-Rod & 0.05 & 0.3 & 0.04 & 0.7 & 0.4 & 250 & 0.7 & 6 & $<0.4$ & 0.4 & 0.2 \\
\hline Map-5B & $<0.05$ & 0.2 & 0.03 & $<0.2$ & $<0.1$ & 115 & 0.6 & 11 & $<0.4$ & 0.3 & 0.9 \\
\hline Map-7 & $<0.05$ & 0.2 & 0.04 & $<0.2$ & $<0.1$ & 227 & 0.5 & 14 & $<0.4$ & $<0.1$ & 0.4 \\
\hline Map-10 & 0.05 & 0.3 & 0.04 & $<0.2$ & $<0.1$ & 48 & 0.2 & 9 & $<0.4$ & 0.3 & 0.9 \\
\hline
\end{tabular}

Turner y Post, 1988), similar a la proporción hallada en la fórmula estructural obtenida en Mina Santa Rosa. De la fórmula general de la romanechita $\left(\mathrm{Ba}, \mathrm{H}_{2} \mathrm{O}\right)_{2}(\mathrm{Mn}, R)_{5}$
$\mathrm{O}_{10}$, donde $R$ puede incluir elementos como $\mathrm{Si}, \mathrm{Al}, \mathrm{Mg}$, $\mathrm{Ca}, \mathrm{Na}, \mathrm{K}, \mathrm{Sr}, \mathrm{W}, \mathrm{Cu}, \mathrm{Zn}$, Ni y Co (Wadsley, 1953; Post, 1999), la fórmula estructural calculada presenta sólo: Al, 


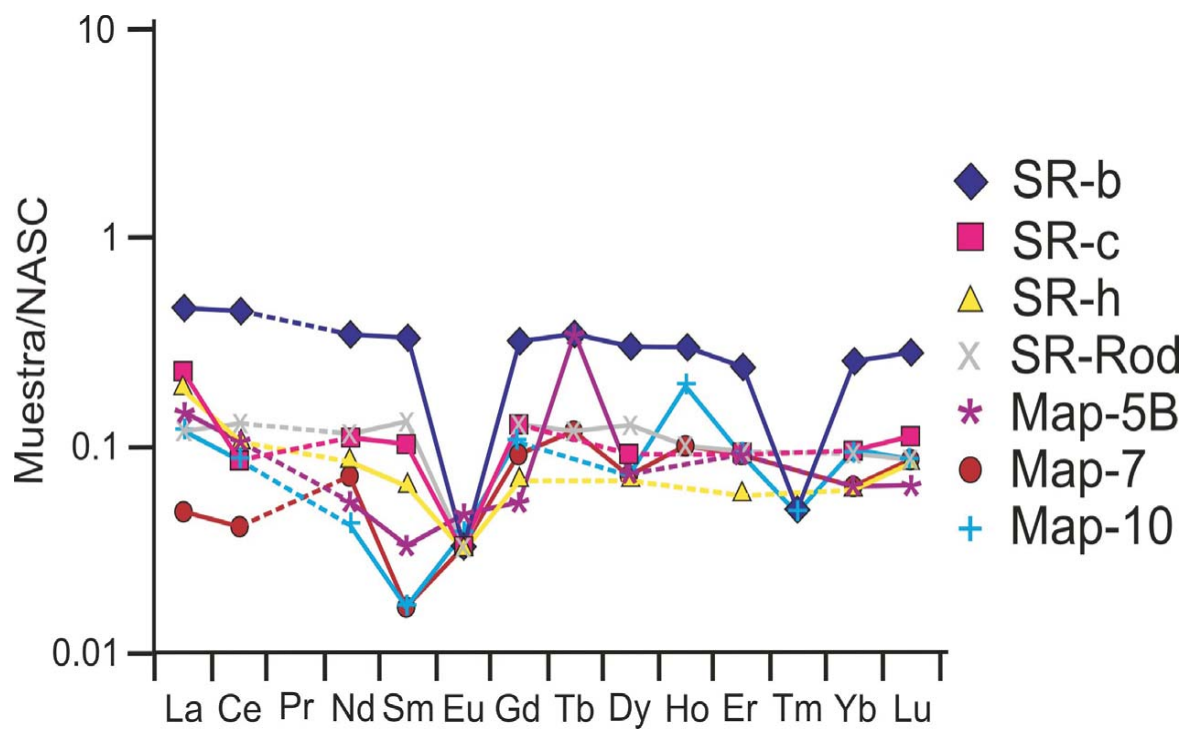

Figura 11. Diagrama de REE normalizadas a NASC (Taylor y McLennan, 1985) de muestras de mena de Santa Rosa (SR) y depósitos del manantial termal Agua Caliente (Map).

$\mathrm{Fe}, \mathrm{Si}, \mathrm{Ca}$ y $\mathrm{Mg}$. Generalmente se encuentra un enriquecimiento distintivo de elementos como $\mathrm{Ba}, \mathrm{Sr}, \mathrm{Ni}, \mathrm{Co}, \mathrm{Cu}$ y $\mathrm{Zn}$ en óxidos de $\mathrm{Mn}$ de depósitos hidrogénicos formados en fondos marinos y un empobrecimiento en los depósitos hidrotermales marinos (Ostwald, 1986; Bonatti et al., 1972; Usui; 1990, Hein et al., 1997). Esta carencia de tales elementos se observa en los óxidos de manganeso de Santa Rosa y Agua Caliente.

La romanechita de los precipitados de los manantiales costeros de Agua Caliente presenta mayor contenido, con respecto a Santa Rosa, en $\mathrm{Si}$, más del $3 \%$ en peso de $\mathrm{SiO}_{2}$, $\mathrm{Mg}$ con más de $1 \%$ en peso de $\mathrm{MgO}$, casi un $2 \%$ en peso de $\mathrm{CaO}$ y $\mathrm{Fe}$ con poco más de $1 \%$ en peso de $\mathrm{Fe}_{2} \mathrm{O}_{3}$. El contenido en $\mathrm{Ba}$ es de 10 wt. \% de $\mathrm{BaO}$ (Canet et al., 2005a), menor a las romanechitas de Mina Santa Rosa.

La paragénesis en Santa Rosa indica una primera etapa de mineralización de romanechita y barita, una segunda etapa de fases silícicas y pirolusita y una tercera supergénica (Figura 8). En los precipitados del manantial termal de Agua Caliente, la mineralización consta de pirita, cinabrio, calcita y barita (Rodríguez-Díaz, 2004; Canet et al., 2005a; Blanco-Florido, 2007). En estos precipitados la barita no se asocia con los óxidos de Mn, pues la romanechita y la todorokita se forman en una segunda etapa de mineralización, y la fase hidrotermal silícica-calcárea ocurre en una etapa tardía.

Es común encontrar las texturas bandeadas coloformes y brechadas en Santa Rosa y en otros depósitos semejantes como en Logan Mine, California, y en Valley, Nuevo México (Mouat, 1962; Hewett, 1964). También en depósitos de manantiales termales se pueden encontrar texturas bandeadas coloformes y travertínicas como en el manantial Golconda, en Nevada (Kerr, 1940) y en Agua Caliente (Canet et al., 2005a, 2005b; Blanco-Florido, 2007). En depósitos de manantial termal es distintiva la formación de sínters silícicos o calcáreos, así como el brechamiento hidrotermal (Berger, 1985). En los depósitos de manantial termal, además, puede haber sulfuros y metales preciosos (Berger, 1985). Estas similitudes con los depósitos del tipo manantial termal pueden observarse entre Santa Rosa y Agua Caliente.

La alteración en el área del depósito Santa Rosa es muy escasa y consiste en un reemplazamiento moderado de los minerales de la roca caja, como plagioclasas y piroxenos, por romanechita, así como una silicificación y oxidación supergénica moderadas, muy similar a lo encontrado en Agua Caliente, en Luis López, Nuevo México (Lueth et al., 2004), en Vani, Milos, Grecia (Liakopoulos et al., 2001) y en Córdoba, Argentina (Leal, 2002), difiriendo únicamente por la presencia de adularia, sericita y motmorillonita, distintivo de yacimientos epitermales (Hedenquist et al., 2000).

\subsection{Fluido mineralizante}

Las mediciones de IF alojadas en barita indican dos poblaciones de IF: una de Th modal de $120^{\circ}-125^{\circ} \mathrm{C}$ y salinidad promedio de $4.2 \mathrm{wt} \% \mathrm{NaCl}$ y otra de Th modal de $110^{\circ}-115^{\circ} \mathrm{C}$ y salinidad promedio de $11.4 w t . \% \mathrm{NaCl}$. Estas poblaciones podrían señalar la existencia de dos fluidos con rangos de temperatura similares pero con variaciones en la salinidad, lo cual es común durante procesos de fraccionamiento composicional producto del proceso de ebullición de los fluidos. Sin embargo, no se han observado evidencias de coatrapamiento de IF salinas y de IF ricas en vapor en las muestras del depósito Santa Rosa, lo cual sería una evidencia de la ocurrencia de ebullición. Esto se puede deber a que en las zonas de sistemas hidrotermales 
con presencia probada de ebullición, se ha observado que solamente el $1 \%$ de la población total de IF son inclusiones ricas en vapor (Simmons y Christenson, 1994), por lo cual se deberían analizar más muestras para aumentar la probabilidad de encontrar inclusiones ricas en vapor. La ebullición es uno de los procesos más efectivos para la precipitación de minerales de mena, en especial minerales preciosos (Simmons y Christenson, 1994).

Una explicación alternativa a los fenómenos de ebullición sería una mezcla en diferentes proporciones de un fluido de baja salinidad con otro de alta salinidad. Las diferentes proporciones de estos fluidos pueden ser el resultado de variaciones temporales de aporte de agua meteórica en el sistema hidrotermal a través de las fallas. En Agua Caliente, datos microtermométricos de IF en barita revelan dos poblaciones con $\mathrm{Th} \sim 127^{\circ} \mathrm{C}$ y una salinidad de $2.7 \mathrm{wt}$. $\% \mathrm{NaCl}$ y otra con $\mathrm{Th}$ de $109^{\circ} \mathrm{C}$ y una salinidad de $11.6 \mathrm{wt}$. $\% \mathrm{NaCl}$ (Villanueva et al., 2008). Estos datos concuerdan con un modelo de mezcla de tres componentes: (a) un fluido meteórico que en su ascenso se mezcla con (b) una salmuera atrapada en una unidad sedimentaria a profundidad (en una contribución entre 20-30\%) y que, eventualmente, en su camino hacia la superficie sufre otra mezcla con (c) agua salina proveniente de infiltraciones someras de agua marina de la Bahía Concepción. Este modelo también puede aplicarse para explicar el fluido mineralizante en Santa Rosa, donde un fluido hidrotermal, posiblemente de origen meteórico, se mezcla con un fluido altamente salino atrapado en unidades sedimentarias miocénicas. Posteriormente, en su asenso a la superficie, el fluido hidrotermal se mezcla en mayor o menor medida con fluidos salinos fríos superficiales de origen marino, motivo por el cual posiblemente se observan variaciones de temperaturas registradas en los datos microtermométricos (Figura 9 y Figura 10). Un moderado rango de Th (en la etapa de brecha) no puede ser provocado sólo por un cambio en la presión ya que en ese caso se tiene una disminución de la temperatura acoplada a un aumento drástico en la salinidad. En los casos de los datos de inclusiones fluidas, se tiene una disminución de la temperatura pero con poca variación en la salinidad, por lo que es factible sugerir un proceso de mezcla.

\subsection{Geoquímica}

La geoquímica de las menas refleja valores de $\mathrm{MnO}$ con un promedio de $40.30 \%$ en peso, concentraciones altas de $\mathrm{SiO}_{2}$ (promedio de $20.63 \%$ en peso), $\mathrm{Ba}(10.63 \%$ en peso) y anomalías de Al, Fe, Ca, Sr, Sb, As, V, W, Cu y Zn (Tabla 3). Los precipitados de los manantiales termales de Agua Caliente presentan concentraciones altas de $\mathrm{SiO}_{2}$ (promedio de $39.5 \%$ en peso), $\mathrm{Al}_{2} \mathrm{O}_{3}$ (promedio 3.7\% en peso) y $\mathrm{Fe}_{2} \mathrm{O}_{3}$ (promedio $1.9 \%$ en peso), con anomalías de $\mathrm{Mn}, \mathrm{Ca}, \mathrm{Sr}, \mathrm{Sb}, \mathrm{V}$ y As. En precipitados de manantiales termales de Hokkaido, en Japón, se puede observar también concentraciones altas de $\mathrm{Mn}$ de entre 23.9 y $59 \%$ en peso
(Miura y Hariya, 1997). Análisis en los lagos relacionados al rifting en el Este de África revelan una depositación por más de 5000 años de sedimentos ricos en $\mathrm{Mn}$, por fluidos hidrotermales con concentraciones de Mn de entre $1.2 \mathrm{ppm}$ hasta $1 \%$ en peso de Mn (Degens y Kulbicki, 1973).

Anomalías de elementos traza como: $\mathrm{Ba}, \mathrm{Sr}, \mathrm{As}, \mathrm{Cu}, \mathrm{Li}$, $\mathrm{Mo}, \mathrm{Sb}, \mathrm{Hg}, \mathrm{Ag}, \mathrm{Au}, \mathrm{Cu}, \mathrm{Pb}, \mathrm{Zn}, \mathrm{Mo}, \mathrm{Ni}$, Co y Tl pueden fungir como indicadores de procesos genéticos hidrotermales de mineralización en yacimientos de manganeso, en especial los que tienen que ver con manantiales termales submarinos y continentales (Hewett, 1964; Ostwald, 1986; Bonatti et al., 1972; Usui, 1990; Nicholson, 1992; Crespo y Lunar, 1997; Hein et al., 1997; Miura y Hariya, 1997; Liakopoulos et al., 2001), tales elementos, más V y W han sido encontrados en el depósito Santa Rosa sugiriendo una génesis hidrotermal relacionada a un manantial, en Agua Caliente también se han encontrado estas anomalías.

Las diferencias marcadas en las concentraciones de Mn y Fe en Santa Rosa y Agua Caliente, denotadas por las relaciones $\mathrm{Fe} / \mathrm{Mn}$, son consecuencia de un drástico fraccionamiento entre estos dos elementos, por condiciones redox y diferencias en su solubilidad, durante la descarga de soluciones hidrotermales en depósitos de tipo manantial termal, como lo observado en Milos, en Grecia (Liakopoulos et al., 2001), acorde con Bonatti (1975), Toth (1980), Nicholson (1992) y Nath et al. (1997). El indicador geoquímico genético de la relación $\mathrm{Co} / \mathrm{Zn}$, señala un carácter hidrotermal para valores de $\mathrm{Co}<<\mathrm{Zn}$ y de origen autigénico Co $>$ Zn (Toth, 1980). En Santa Rosa y Agua Caliente se encontraron valores de $\mathrm{Co}<<\mathrm{Zn}$, entre 0.173 y 0.338 y 0.029 a 0.267 , respectivamente, lo que indica un carácter genético hidrotermal para Santa Rosa y Agua Caliente.

Con respecto a las concentraciones totales de LREE y HREE en Santa Rosa y Agua Caliente (Tabla 3), se puede observar una mayor concentración de LREE para ambos casos. Tal predominancia en LREE con respecto a HREE puede ser explicada por una génesis hidrotermal (Nath et al., 1997), por lo que se presume un origen hidrotermal para Santa Rosa y Agua Caliente.

En los espectros de REE normalizados a NASC en Santa Rosa se puede observar una anomalía negativa de $\mathrm{Eu}$, $\left(\mathrm{Eu} / \mathrm{Eu}^{*}=0.017\right.$ a 0.030$)$ y una anomalía negativa menos marcada de $\mathrm{Ce}(\mathrm{Ce} / \mathrm{Ce} *=0.074$ a 0.123$)$. Las muestras de Agua Caliente presentan una pequeña anomalía negativa de $\mathrm{Ce}\left(\mathrm{Ce} / \mathrm{Ce}^{*}=0.053\right.$ a 0.112$)$, muy similar a la de Santa Rosa (Figura 11).

Dentro de las REE el Eu es significativamente móvil en fluidos hidrotermales (Michard et al., 1983). La movilidad del Eu está regida por las condiciones redox, donde un empobrecimiento en $\mathrm{Eu}$ indica fluidos hidrotermales oxidados y de baja temperatura, y un enriquecimiento en Eu revela fluidos calientes y reducidos (Parr, 1992). La anomalía negativa de Eu en las menas de Santa Rosa denota fluidos oxidados y de baja temperatura, coincidiendo con una mayor presencia de óxidos de manganeso que de bari- 
ta, mientras que en Agua Caliente se reportan anomalías ligeramente positivas de Eu (Canet et al., 2005a), indicando fluidos ligeramente reducidos y de moderada temperatura, concordando con una mayor presencia de barita, pirita y cinabrio que de óxidos. Una anomalía negativa de $\mathrm{Ce}$ es una característica distintiva para depósitos hidrotermales de Fe-Mn (Nath et al., 1997), coincidente con lo encontrado en el depósito de Santa Rosa y en Agua Caliente. El empobrecimiento en $\mathrm{Ce}$ en un depósito hidrotermal, puede atribuirse a la oxidación de $\mathrm{Ce}^{3+}$ a $\mathrm{Ce}^{4+}$ en agua marina y a una subsecuentemente removilización (Nath et al., 1997; Alibo y Nozaki, 1999). Las concentraciones moderadamente bajas en Ce y las salinidades obtenidas en IF en Santa Rosa y Agua Caliente sugieren que son fluidos salinos en condiciones oxidantes los causantes de la mineralización. Villanueva et al. (2008) a través de un modelado geoquímico menciona una mezcla de fluidos meteóricos, salmueras profundos y agua de mar como fluidos causantes de la mineralización en Agua Caliente, modelo compatible con los datos geoquímicos y microtermométricos.

\section{Conclusiones}

La mineralización de Mn-Ba de Santa Rosa presenta muchas afinidades con los adyacentes manantiales termales costeros de Agua Caliente, como son: (a) las estructuras de tipo veta, con orientación NNO-SSE controladas por fallas normales del Mioceno Superior-Plioceno, (b) las asociaciones minerales, ricas en romanechita, barita y ópalo, principalmente, con relaciones texturales y paragenéticas comparables, (c) la firma geoquímica, caracterizada por el enriquecimiento en $\mathrm{Mn}, \mathrm{Ba}, \mathrm{Sb}, \mathrm{As}, \mathrm{Cu}$ y $\mathrm{Zn}$, diferencias marcadas en las concentraciones de $\mathrm{Mn}$ y $\mathrm{Fe}(\mathrm{Fe} / \mathrm{Mn})$ y Co y $\mathrm{Zn}(\mathrm{Co} / \mathrm{Zn})$ y (d) los fluidos mineralizantes salinos en condiciones oxidantes, de baja temperatura, $97^{\circ}$ a $160^{\circ} \mathrm{C}$, y salinidades altas, $\sim 4$ y de $11.1 w t . \% \mathrm{NaCl}$.

Ambas mineralizaciones guardan una estrecha relación metalogenética, pudiendo considerarse a la mineralización en Santa Rosa como un equivalente fósil del manantial termal de Agua Caliente.

En ambos casos, el sistema hidrotermal está ligado a la tectónica extensional, de modo que un fluido mayoritariamente meteórico circula por sistemas de fallas normales que en su ascenso sufre una mezcla con fluidos salinos. En su recorrido hacia la superficie, el fluido hidrotermal se enriquece en $\mathrm{Mn}, \mathrm{Ba}, \mathrm{Si}, \mathrm{Fe}, \mathrm{Ca}, \mathrm{Sr}, \mathrm{Sb}$ y As, como resultado de la interacción con los estratos subyacentes. Los procesos de mineralización podrían ser originados por una ebullición y/o por mezcla de fluidos.

En consecuencia, el depósito de Mn-Ba de Santa Rosa constituye un caso de mineralización hidrotermal de baja temperatura en vetas extensionales, formado en un ambiente muy cercano a la superficie, de manantial termal (hot-spring).

\section{Agradecimientos}

La investigación que ha resultado en este trabajo ha sido financiada por el proyecto J-51127-I (CONACyT). Agradecemos a Rufino Lozano Santa Cruz (Instituto de Geología, UNAM) su apoyo en el laboratorio de FRX, a Carlos Linares López y Margarita Reyes (Laboratorio Universitario de Petrología, Instituto de Geofísica, UNAM) y a María Guadalupe Dávalos Elizondo (Posgrado en Ciencias de la Tierra, UNAM) por sus comentarios acerca de los datos de EMP y SEM. Agradecemos por sus valiosos consejos en la interpretación y discusión de los resultados de DRX e IF a E. Tauler Ferré y S. Martínez Manent (UB), R. E. Villanueva y A. Camprubí (UNAM).

Deseamos agradecer también a A. Camprubí, R.G. Pérez Vázquez, C. Báncora y A. López-Sánchez su apoyo durante diversas salidas de campo a la zona de estudio.

Los autores agradecemos a Lorena Ortega (Universidad Complutense de Madrid), Rafael Del Rio Salas (Universidad de Arizona) y Luis Enrique Ortiz Hernández (Universidad Autónoma del Estado de Hidalgo) por sus exhaustivas revisiones y valiosos comentarios de este trabajo.

\section{Bibliografía}

Alibo, D.S., Nozaki, Y., 1999, Rare earth elements in seawater: particle association, shale normalization, and Ce oxidation: Geochimica et Cosmochimica Acta, 63, 363-372.

Berger, B.R., 1985, Geological-geochemical features of hot-spring precious metal deposits, in Tooker, E. W. (ed.), Geological characteristics of sediments and volcanic hosted disseminated gold deposits- Search for an occurrence model: U.S. Geological Survey Bulletin, 1646, 47-54.

Bigioggero, B., Chiesa, S., Zanchi, A., Montrasio, A., Vezzoli, L., 1995, The Cerro Mencenares Volcanic Center, Baja California Sur: Source and tectonic control on postsubduction magmatism within the Gulf Rift: Geological Society of America Bulletin, 107, 1108-1122.

Blanco-Florido, D., 2007, Caracterización de las mineralizaciones asociadas a manantiales hidrotermales costeros en Bahía Concepción, Baja California Sur, México: México, D. F., México, Universidad Nacional Autónoma de México, Facultad de Ingeniería, tesis de licenciatura, $55 \mathrm{p}$.

Bodnar, R.J., 1983, A method of calculating fluid inclusion volumes based on vapor bubble diameters and P-V-T-X properties of inclusion fluids: Economic Geology, 78, 535-542.

Bodnar, R. J., 1993, Revised equation and table for determining the freezing point depression of $\mathrm{H}_{2} \mathrm{O}-\mathrm{NaCl}$ solutions: Geochimica et Cosmochimica Acta, 57, 683-684.

Bonatti, E., 1975, Metallogenesis at oceanic spreading centers: Annual Review of Earth and Planetary Sciences, 3, 401-431.

Bonatti, E., Kramer, T., Rydell, H., 1972, Classification and genesis of submarine iron-manganese deposits, in Horn, D.R. (ed.), Ferromanganese deposits of the Ocean Floor: Washington, National Science Foundation, 147-166.

Bursill, L.A., 1979, Structural Relationships Between $\beta$-Gallia, Rutile, Hollandite, Psilomelane, Ramsdellite and Gallium Titanate Type Structures: Acta Crystallogaphica, B35, 530-538.

Camprubí, A., Canet, C., Rodríguez-Díaz, A.A., Prol-Ledesma, R.M., Blanco-Florido, D., Villanueva, R.E., López-Sánchez, A., 2008, Geology, ore deposits and hydrothermal venting in Bahía Concepción, Baja California Sur, Mexico: Island Arc, 17, 6-25. 
Canet, C., Prol-Ledesma, R.M., Proenza, J.A., Rubio-Ramos, M.A., Forrest, M.J., Torres-Vera, M.A., Rodríguez-Díaz, A.A., 2005a, $\mathrm{Mn}-\mathrm{Ba}-\mathrm{Hg}$ mineralization at shallow submarine hydrothermal vents in Bahía Concepción, Baja California Sur, Mexico: Chemical Geology, 224, 96-112.

Canet, C., Prol-Ledesma, R.M., Torres-Alvarado, I., Gilg, A.H., Villanueva, R.E., Lozano-Santa Cruz, R., 2005b, Silica-carbonate stromatolites related to coastal hidrotermal venting in Bahía Concepción, Baja California Sur, México: Sedimentary Geology, 174, 97-113.

Canet, C., Prol-Ledesma, R. M., Bandy, W.L., Schaaf, P., Linares, C. Camprubí, A., Tauler, E., Mortera-Gutiérrez, C., 2008, Mineralogical and geochemical constraints on the origin of ferromanganese crust from the Rivera Plate (western margin of Mexico): Marine Geology, 251, 47-59.

Casarrubias, U.Z., Gómez-López, G., 1994, Geología y evaluación geotérmica de la zona de Bahía Concepción, Baja California Sur, México (resumen), en $3^{\mathrm{a}}$ Reunión Internacional sobre geología de la Península de Baja California: La Paz, B. C. S., México, Universidad Autónoma de Baja California Sur, 22-23.

Crespo, A., Lunar, R., 1997, Terrestrial hot- spring Co-rich Mn mineralization in the Pliocene-Quaternary Calatrava Region (central Spain), in Nicholson, K., Hein, J. R., Buhn, B., Dasgupta, S. (eds.), Manganese mineralization, geochemistry and mineralogy of terrestrial and marine deposits: London, Geological Society Special Publication, 119, 253-264.

Degens, E.T., Kulbicki, G., 1973, Hydrothermal origin of metals in some East African Rift Lakes: Mineral Deposita, 8, 388-404.

Del Rio-Salas, R., Ruiz, J., Ochoa-Landín, L., Noriega, O., Barra, F., Meza-Figueroa, D., Paz-Moreno, F., 2008, Geology, Geochemistry and Re-Os systematics of manganese deposits from the Santa Rosalía Basin and adjacent areas in Baja California Sur, México: Mineralium Deposita, 43, 467-487.

Freiberg, D.A., 1983, Geologic Setting and Origin of the Lucifer Manganese Deposits, Baja California Sur, Mexico: Economic Geology, 78, 931-943.

González-Reyna, J., 1956, Los Yacimientos de Manganeso de El Gavilán, Azteca y Guadalupe, Baja California, México, en González-Reyna (ed.), Symposium del Manganeso. XX Congreso Geológico Internacional: México, D. F., UNAM, III, 79-96.

Hausback, B.P., 1984, Cenozoic volcanic and tectonic evolution of Baja California, Mexico, in Frizzell, V.A. (ed.), Geology of the Baja California Peninsula, Field Trip Guidebook: San Diego, E.U.A., Society of Economic Paleontologists and Mineralogists, Pacific Section, 39, 219-236.

Hedenquist, J.W., Arribas, A. Jr., Urien-Gonzalez, E., 2000, Exploration for epithermal gold deposits, in Hagemann, S.G., Brown, P.E. (eds.), Gold in 2000: Society of Economic Geologists, Reviews in Economic Geology, 13, 245-277.

Hein, J.R., Koschinsky, A., Halbach, P., Manheim, F. T., Bau, M., Kang, J.K., Lubick, N., 1997, Iron and manganese oxide mineralization in the Pacific, in Nicholson, K., Hein, J.R., Buhn, B., Dasgupta. S. (eds.), Manganese mineralization, geochemistry and mineralogy of terrestrial and marine deposits: London, Geological Society Special Publication, 119, 123-138.

Herdianita, N.R., Browne, P.R.L., Rodgers, K.A., Campbell, K.A., 2000, Mineralogical and morphological changes accompanying aging of siliceous sinter and silica residue: Mineralium Deposita, 35, 48-62.

Hewett, D.F., 1964, Veins of hypogene manganese oxide minerals in the southwestern United States: Economic Geology, 59, 1429-1472.

Johnson, M.E., Ledesma-Vázquez, J., Mayall, M.A., Minch, J., 1997, Upper Pliocene stratigraphy and depositional systems: the Peninsula Concepción Basin in Baja California Sur, Mexico, in Johnson, M.E., Ledesma-Vázquez, J. (eds.) Pliocene Carbonates and Related Facies Flanking the Gulf of California, Mexico: Geological Society of America Special Papers, 318, 57-72.

Johnson, M.E., Ledesma-Vázquez, J., 2001, Pliocene-Pleistocene rocky shorelines trace coastal development of Bahía Concepción, gulf coast of Baja California Sur (Mexico): Palaeogeography, Palaeoclimatology, Palaeoecology, 166, 65-88.
Kerr, P.F., 1940, Tungsten-bearing manganese deposits at Golconda, Nevada: Geological Society of America Bulletin, 51, 1359-1390.

Laznicka, P., 1992, Manganese deposits in the global lithogenetic system: Quantitative approach: Ore Geology Reviews, 7, 279-356.

Leal, P.R., 2002, Inclusiones fluidas e isótopos estables en la ganga de los yacimientos de manganeso del norte de la provincia de Córdoba: Revista de la Asociación Geológica Argentina, 57, 251-259.

Ledesma-Vázquez, J., 2000, Cuencas sedimentarias del Plioceno en el Golfo de California; Cuenca San Nicolás, Baja California Sur: La Paz, México, Universidad Autónoma de Baja California, Facultad de Ciencias Marinas, Instituto de Investigaciones Oceanológicas, tesis doctoral, $116 \mathrm{p}$.

Ledesma-Vázquez, J., Johnson, M.E., 2001, Miocene-Pleistocene tectono-sedimentary evolution of Bahía Concepción region, Baja California Sur (México): Sedimentary Geology, 144, 83-96.

Ledesma-Vázquez, J., Berry, R.W., Johnson, M.E., Gutiérrez-Sánchez, S., 1997, El Mono chert: a shallow-water chert from the Pliocene Infierno Formation, Baja California Sur, Mexico: Geological Society of America Special Paper, 318, 73-81.

Ledesma-Vázquez, J., Carreño, A.L., Staines-Urias, F., Johnson, M.E., 2006, The San Nicolás Formation: A Proto-Gulf ExtensionalRelated New Lithostratigraphic Unit at Bahía San Nicolás, Baja California Sur, Mexico: Journal of Coastal Research, 22, 801-811. Liakopoulos, A., Glasby, J.P., Papavassiliou , C.T., Boulegue, J., 2001, Nature and origin of the Vani manganese deposit, Milos, Greece: an overview: Ore Geology Reviews, 18, 181-209.

Lueth, V.W., Chamberlin, R.M., Peters, L., 2004, Age of mineralization in the Luis Lopez manganese district, Socorro County, New Mexico, as determined by ${ }^{40} \mathrm{Ar} /{ }^{39} \mathrm{Ar}$ dating of crytomelane: New Mexico Bureau of Geology \& Mineral Resources Bulletin 160, 239-249.

Lynne, B.Y., Campbell, K.A., Moore, J. N., Browne, P.R.L., 2005, Diagenesis of 1900-year-old siliceous sinter (opal-A to quartz) at Opal Mound, Roosevelt Hot Springs, Utah, U.S.A.:, Sedimentary Geology 179, 249-278.

Mapes, V.E., 1956, El Manganeso en México, en González-Reyna (ed.), Symposium del Manganeso, XX Congreso Geológico Internacional: México D.F., México, UNAM, III, 35-74.

Maraver-Romero, D.A., Aguilera-Hale, J.C., Sevilla-Unda, V.E., Meléndez-Rodríguez, A., 1997, Carta Geológica Minera Loreto G12-5, escala 1:250 000: Pachuca, Hidalgo, México, Consejo de Recursos Minerales, 1 mapa.

Mouat, M.M., 1962, Manganese oxides from The Artillery Mountains Area, Arizona: The American Mineralogist, 47, 744-757.

McFall, C.C., 1968, Reconnaissance Geology of the Concepcion Bay area, Baja California, Mexico: Stanford University Publications Geological Sciences, 10, 1-25.

McLennan, S.M., 1989, Rare Earth Elements in sedimentary rocks: influence of provenance and sedimentary processes: Reviews in Mineralogy and Geochemistry, 21, 169-200.

Michard, A., Albarède, F., Michard, G., Minster, J.F., Charlou, J.L., 1983, Rare-earth elements and uranium in high-temperature solutions from East Pacific Rise hydrothermal vent field $\left(13^{\circ} \mathrm{N}\right)$ : Nature, 303, 795-797.

Miura, H., Hariya, Y., 1997, Recent manganese oxide deposits in Hokkaido, Japan, in Nicholson, K., Hein, J. R., Bühn, B., Dasgupta, S. (eds.), Manganese mineralization, geochemistry and mineralogy of terrestrial and marine deposits: London, Geological Society Special Publication, 119, 281-299.

Nath, N., Plüger, W., Roelands, I., 1997, Geochemical constraints on the hydrothermal origin of ferromanganese encrustations from the Rodriguez Triple Junction, Indian Ocean, in Nicholson, K., Hein, J. R., Bühn, B., Dasgupta, S. (eds.), Manganese mineralization, geochemistry and mineralogy of terrestrial and marine deposits: London, Geological Society Special Publication,, 119, 199-212.

Nicholson, K., 1992, Contrasting mineralogical-geochemical signatures of manganese oxides: guides to metallogenesis: Economic Geology, 87, 1253-1264.Noble, J.A., 1950, Manganese on Punta Concepcion, Baja California, Mexico: Economic Geology, 45, 771-785.

Ostwald, J., 1986. Some observations in the chemical composition of todorokite: Mineralogical Magazine, 50, 336-340. 
Parr, J.M., 1992, Rare-earth element distribution in exhalites associated with Broken Hill-type mineralisation at the Pinnacles Deposit, New South Wales, Australia: Chemical Geology, 100, 73-91.

Post, J.E., 1999, Manganese oxide minerals: Crystal structures and economic and environmental significance, Proceedings of the National Academy of Science of the Unites States of America, 96, 3447-3454.

Prol-Ledesma, R.M., Canet, C., Torres-Vera, M.A., Forrest, M.J., Armienta, M.A., 2004, Vent fluid chemistry in Bahía Concepción coastal submarine hydrothermal system, Baja California Sur, Mexico: Journal of Volcanology and Geothermal Research, 137, 311-328.

Roedder, E., 1984, Fluid Inclusions, in Ribbe, P.H. (ed.), Fluid Inclusions: Reviews in Mineralogy 12, 644.

Rodríguez-Díaz, A.A., 2004, Caracterización geológica y geoquímica del área mineralizada de manganeso en Bahía Concepción, Baja California: México D. F., México, Universidad Nacional Autónoma de México, Facultad de Ingeniería, tesis de licenciatura, 90 p.

Roy, S., 1992, Environments and processes of manganese deposition: Economic Geology, 87, 1218-1236.

Roy, S., 1997, Genetic diversity of manganese deposition in the terrestrial geological record, in Nicholson, K., Hein, J. R., Bühn, B., Dasgupta, S. (eds.), Manganese mineralization, geochemistry and mineralogy of terrestrial and marine deposits: London, Geological Society Special Publication, 119, 5-27.

Simmons, S.F., Christenson, B.W., 1994, Origins of calcite in a boiling geothermal system: American Journal of Science, 294, 361-400.

Smith, D.K., Mrose M.E., 1974, Selected Powder Diffraction Data for Minerals, Joint Committee on Powder Diffraction Standards, 1-4.

Swalan, M.G., Smith, J.G., 1984, Petrologic characteristics, age and tectonic setting of Neogene volcanic rocks in northern Baja California Sur, México, in Frizell, V.A. Jr. (ed.), Geology of the Baja California Peninsula: Los Angeles, E.U.A., Society of Economic Paleontologits and Mineralogist, Pacific Section, Book 39, 237-251.

Taylor, S.R., McLennan, S.M., 1985, The Continental Crust: Its Composition and Evolution: Surrey, U.K., Blackwell Scientific Publications, $312 \mathrm{p}$.

Terán-Ortega, L.A., Ávalos-Zermeño, A., 1993, Informe de definición del prospecto Las Mantitas, Anomalía Pilares, Área Bahía Concepción, Zona El Azufre, Municipio de Mulegé, Estado de Baja California Sur: La Paz, Baja California Sur, Consejo de Recursos Minerales, Informe Técnico, $65 \mathrm{p}$.

Toth , J.R., 1980, Deposition of submarine crust rich in manganese and iron: Geological Society of America Bulletin, 91,. 44.-54.

Turner, S., Buseck, P.R., 1981, Todorokites: a new family of natural occurring manganese oxides: Science, 212, 1024-1027.
Turner, S., Post, J.E., 1988, Refinement of the substructure and superstructure of romanechite: American Mineralogist, 73, 1155-1161.

Ulrich, M.R., Bodnar, R.J., 1988, Systematics of stretching of fluid inclusions II; Barite at 1 atm confining pressure: Economic Geology, 83, 1037-1046.

Umhoefer, P.J., Dorsey, R.J., Willsey, S., Mayer, L., Renne, P., 2001, Stratigraphy and geochronology of the Comondú Group near Loreto, Baja California Sur, Mexico: Sedimentary Geology, 144, 125-147.

Umhoefer, P.J., Mayer, L., Dorsey, R.J., 2002, Evolution of the margin of the Gulf of California near Loreto, Baja California Peninsula, Mexico: Geological Society of America Bulletin, 114, 849-868.

Usui, A., 1990, Deposition of hydrogenetic and hydrothermal manganese minerals in the Bonin Arc area, West Pacific (abstract), in Fifth Circum-Pacific Energy and Mineral Resources Conference: Honolulu, Hawaii, U.S.A, AAPG, 1007.

Villanueva, E.R.E., Prol-Ledesma, R.M., Rodríguez-Díaz, A.A., González-Partida, E., Canet, C., Torres-Alvarado, I. S., 2009, Possible origin of shallow submarine hydrothermal fluids using mixing geochemical modeling: Bahía Concepción, Baja California Sur, México: Journal of Volcanology and Geothermal Research, aceptado.

Van den Kerkhof, A.M., Hein, U.F., 2001, Fluid inclusion petrography: Lithos, 55, 27-47.

Vasconcelos, P.M., 1999, K-Ar and 40Ar/39Ar Geochronology of weathering processes: Annual Reviews of Earth and Planetary Sciences, 27, 183-229.

Wadsley, A.D., 1953, The Crystal Structure of Psilomelane, (Ba, $\left.\mathrm{H}_{2} \mathrm{O}\right)_{2} \mathrm{Mn}_{5} \mathrm{O}_{10}$ : Acta Crystallographica 6, 433-438.

White, D.E., 1981, Active geothermal systems and hydrothermal ore deposits: Economic Geology, $75^{\text {th }}$ Anniversary Volume, 392-423.

Zantop, H., 1978, Geological setting and genesis of iron oxides and manganese oxides in the San Francisco manganese deposit, Jalisco Mexico: Economic Geology, 73, 1137-1149.

Zhang, Y., Frantz, J.D., 1987, Determination of the homogenization temperatures and densities of supercritical fluids in the system $\mathrm{NaCl}-\mathrm{KCl}-\mathrm{CaCl} 2-\mathrm{H} 2 \mathrm{O}$ using synthetic fluid inclusions: Chemical Geology, 64, 335-350.

Recibido: 02/10/2008

Recibido corregido: 28/11/2008

Aceptado: 10/12/2008 